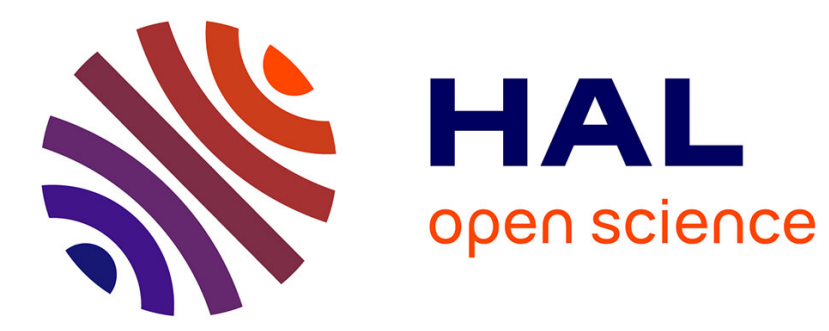

\title{
Price of Anarchy in Non-Cooperative Load Balancing
} Urtzi Ayesta, Olivier Brun, Balakrishna Prabhu

\section{To cite this version:}

Urtzi Ayesta, Olivier Brun, Balakrishna Prabhu. Price of Anarchy in Non-Cooperative Load Balancing. 2009. hal-00416123

\section{HAL Id: hal-00416123 \\ https://hal.science/hal-00416123}

Preprint submitted on 11 Sep 2009

HAL is a multi-disciplinary open access archive for the deposit and dissemination of scientific research documents, whether they are published or not. The documents may come from teaching and research institutions in France or abroad, or from public or private research centers.
L'archive ouverte pluridisciplinaire HAL, est destinée au dépôt et à la diffusion de documents scientifiques de niveau recherche, publiés ou non, émanant des établissements d'enseignement et de recherche français ou étrangers, des laboratoires publics ou privés. 


\title{
Price of Anarchy in Non-Cooperative Load Balancing
}

\author{
U. Ayesta, O. Brun, B.J. Prabhu \\ LAAS-CNRS \\ Université de Toulouse \\ 7 Avenue du Colonel Roche \\ 31077 Toulouse, France. \\ email: \{urtzi, brun, bjprabhu\}@laas.fr
}

\begin{abstract}
We investigate the price of anarchy of a load balancing game with $K$ dispatchers. The service rates and holding costs are assumed to depend on the server, and the service discipline is assumed to be processor-sharing at each server. The performance criterion is taken to be the weighted mean number of jobs in the system, or equivalently, the weighted mean sojourn time in the system.

We first show that, for a fixed amount of total incoming traffic, the worst-case Nash equilibrium occurs when each player routes exactly the same amount of traffic, i.e., when the game is symmetric. For this symmetric game, we provide the expression for the loads on the servers at the Nash equilibrium. Using this result we then show that, for a system with two or more servers, the price of anarchy, which is the worst-case ratio of the global cost of the Nash equilibrium to the global cost of the centralized setting, is lower bounded by $K /(2 \sqrt{K}-1)$ and upper bounded by $\sqrt{K}$, independently of the number of servers.
\end{abstract}

\section{Introduction}

Server farms are used nowadays in as diverse areas as e-service industry, database systems and grid computing clusters. Figure 1 depicts the typical architecture of a server farm with a single centralized dispatcher who receives jobs from different sources and routes them to a set of servers. Server farms have become a popular architecture in computing centers and are used, for example, in the Cisco Local Director, IBM Network Dispatcher and Microsoft Sharepoint (see [4] for a recent survey). This configuration can also be used to model a web server farm, where requests for files (or HTTP pages) arrive to a dispatcher and are dispatched immediately to one of the servers in the farm for processing. 


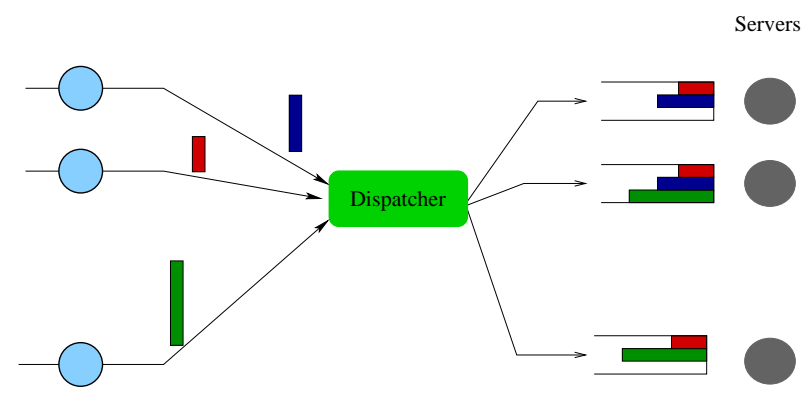

Figure 1: Centralized architecture for a server farm.

One of the fundamental issues in this context is to characterize the optimal routing strategy. The problem amounts to finding the routing strategy of the dispatcher that will optimize a certain performance objective such as the mean processing time (or sojourn time) of jobs. By Little's law, this performance objective is equivalent to the mean number of jobs in the system. Such a routing strategy is known as the social optimum or the social welfare since it minimizes the mean processing time of jobs (we will also refer to it as the global optimum). This load balancing problem is perhaps one of the most studied one in the operations research community, and many works have been devoted to the analysis of the optimal routing in various static and dynamic scenarios $[9,13,20]$.

In practice, it may however happen that a single centralized dispatcher is simply not feasible due to scalability or complexity reasons. In this case, the system designer will certainly have to resort to a distributed scheme in which several dispatchers are used as shown in Figure 2. In this case, each dispatcher will independently seek to minimize the processing time perceived by the traffic it routes. Thus, the shift from a centralized to a distributed scheme will give rise to a non-cooperative game between the dispatchers.

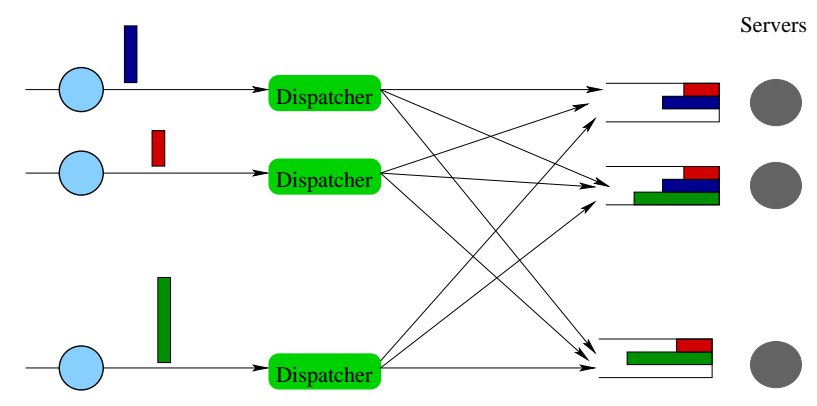

Figure 2: Decentralized architecture for a server farm. 
Game theory provides the systematic framework to study and to understand such problems. We can distinguish two different types of games depending on the number of dispatchers. If the number of dispatchers is finite then the game is said to be "atomic" and a well-known equilibrium strategy is given by the Nash equilibrium, that is, a routing strategy from which unilateral deviation does not help any dispatcher in improving the performance perceived by the traffic it routes. If the number of dispatchers is infinite (every arriving job takes its own routing decision and can be thought of as a dispatcher) the game is said to be "non-atomic" and the corresponding equilibrium is given by the notion of Wardrop equilibrium. In this case, the equilibrium point is characterized by the fact that the performance in every (used) server is the same. In the present article we are mostly interested in the "atomic" setting, and we refer to Section 2 for related work in the "non-atomic" setting.

From the system designer's perspective a very important question pertains to the loss of performance incurred when shifting to a decentralized architecture. Indeed, in the decentralized architecture each dispatcher performs an individual optimization for its own jobs, and thus it can be expected that the overall performance of the decentralized scheme will be worse than that of the centralized scheme. The system designer is probably ready to accept a distributed routing scheme provided that the gain in scalability is not achieved at the expense of a significant loss in performance. In this context, the question turns out to be: can we provide performance guarantees for these decentralized routing schemes? This is the main question addressed in this paper.

Our objectives are two fold. Firstly, we investigate the properties of the noncooperative game. We show that there always exists a unique Nash equilibrium, that is, a routing strategy from which no dispatcher has any incentive to deviate. We also show that the worst Nash equilibrium occurs when the amount of traffic that every dispatcher routes is exactly the same. To the best of our knowledge this property has not been shown previously, and it may find applications in other games. For this particular case, we show that the game belongs to a particular class of games known as Potential Games [16] which are known to have several desirable properties. For instance, for a potential game, the best response algorithm converges to the equilibrium. Secondly, we compare the performance of the global optimum with that given by the Nash equilibrium. In other words, we compare the performance when there is only one dispatcher which routes all the traffic with the performance when there are several dispatchers each one seeking to optimize its own performance. In order to do so we look at the Price of Anarchy (PoA) which was introduced by Koutsoupias and Papadimitriou [15]. The PoA is a measure of the inefficiency of a decentralized scheme. It is defined as the ratio of the performance obtained by the worst Nash equilibrium to that of the global optimal solution, and hence it lies in the interval $[1, \infty)$. We show that the PoA is of the order of the square root of the number of dispatchers. Thus, it grows fairly quickly and unboundedly with the number of dispatchers. As a consequence, we recover the result in [1] where it was shown that when the number of dispatchers is infinite (the "non-atomic" as pointed out above) the 
PoA is infinite.

The rest of the paper is organized as follows. In Section 3, we describe the model and state the problem. In Section 4, we explore the structure of the underlying Nash equilibria and prove their existence and uniqueness. We also establish several properties of these equilibria that form the foundation of the subsequent analysis. In Section 5, we analyze the global cost at the Nash equilibria, and show that the maximum of this cost is achieved in the symmetric case. With this result at hand, in Section 6, we derive lower and upper bounds on the PoA. Finally, we draw some conclusions and give possible extensions in Section 7 .

\section{Related work}

Load balancing in multi-server systems has been widely studied in the literature. Global and individual optimality in load balancing are considered in the monograph [13], which does not consider decisions based on the knowledge of the amount of load. Systems with a general service-time distribution and the FCFS scheduling discipline were studied in $[6,2,3,10]$, while [17, 12] studied systems with an exponential service-time distributions and an arbitrary scheduling discipline. In [11], the authors analyzed a multi-server system where requests join the server that has the smallest number of requests. In a recent work [5], the authors investigate the performance of a server farm where the scheduling discipline in each server is SRPT (Shortest Remaining Processing Time First). In [8], the authors studied the performance of selfish routing in a server farm with a min-max objective, that is, when the objective is to minimize the maximum sojourn time in the servers.

In recent years the study of PoA in multi-server queues has started to receive attention. In [12], the authors considered the non-atomic scenario where every arriving job can select the server in which it will be served. An important assumption is that the holding cost is the same in every server. Building upon results from [2], it is shown in [12] that the PoA is upper bounded by the number of servers. We also refer to [21] for similar results. Another closely related work is [1]. The main difference between the models studied in [12] and [1] is that in the latter the holding costs in every server could be arbitrarily chosen. Using potential game theory, it is shown in [1] that the PoA is unbounded in the nonatomic setting, i.e., it can be arbitrarily close to infinity. This was a surprising result since it indicated that unequal holding costs may have a profound impact on the system's performance.

Our present work is closely related to work by Orda and co-authors [14, 18]. In these references the atomic non-cooperative setting was studied, but the focus was on existence, uniqueness and the properties of the Nash equilibrium rather than on the PoA. Moreover, it was also assumed that the holding cost per unit 
of time is the same in every server, which as we have mentioned can have a profound impact on the performance. Several of the arguments used in the present work are directly inspired from those references, but we emphasize that our main results and characterizations are new.

\section{Problem Formulation and Main Results}

We consider a non-cooperative routing game with $K$ dispatchers and $S$ ProcessorSharing servers. Denote $\mathcal{C}=\{1, \ldots, K\}$ to be the set of dispatchers and $\mathcal{S}=\{1, \ldots, S\}$ to be the set of servers. Jobs received by dispatcher $i$ are said to be jobs of class $i$.

Server $j \in \mathcal{S}$ has capacity $r_{j}$ and a holding cost $c_{j}$ per unit time is incurred for each job sent to this server. It is assumed that servers are numbered in the order of increasing cost per unit capacity, i.e., if $m \leq n$, then $\frac{c_{m}}{r_{m}} \leq \frac{c_{n}}{r_{n}}$. Let $\mathbf{r}=\left(r_{j}\right)_{j \in \mathcal{S}}$ and $\mathbf{c}=\left(c_{j}\right)_{j \in \mathcal{S}}$ denote the vectors of server capacities and server costs, respectively, and let $\bar{r}=\sum_{n \in \mathcal{S}} r_{n}$ denote the total capacity of the system.

Jobs of class $i \in \mathcal{C}$ arrive to the system according to a Poisson process and have generally distributed service-times. We do not specify the arrival rate and the characteristics of the service-time distribution due to the fact that in an $M / G / 1-P S$ queue the mean number of jobs depends on the arrival process and service-time distribution only through the traffic intensity, i.e., the product of the arrival rate and the mean service-time.

Let $\lambda_{i}$ be the traffic intensity of class $i$. It is assumed that $\lambda_{i} \leq \lambda_{j}$ for $i \leq j$. Moreover, it will also be assumed that the vector $\boldsymbol{\lambda}$ of traffic intensities belongs to the following set:

$$
\Lambda=\left\{\lambda \in \mathbb{R}^{K}: \sum_{i \in \mathcal{C}} \lambda_{i}=\bar{\lambda}\right\}
$$

where $\bar{\lambda}$ denotes the total incoming traffic intensity. It will be assumed throughout the paper that $\bar{\lambda}<\bar{r}$, which is the necessary and sufficient condition to guarantee the stability of the system.

Let $\mathbf{x}_{i}=\left(x_{i, j}\right)_{j \in \mathcal{S}}$ denote the routing strategy of dispatcher $i$, with $x_{i, j}$ being the amount of traffic it sends towards server $j$. Let

$$
\mathcal{X}_{i}=\left\{\mathbf{x}_{i} \in \mathbb{R}^{S}: 0 \leq x_{i, j} \leq r_{j}, \forall j \in \mathcal{S} ; \sum_{j \in \mathcal{S}} x_{i, j}=\lambda_{i}\right\}
$$

denote the set of feasible routing strategies for dispatcher $i$. The vector $\mathbf{x}=$ $\left(\mathbf{x}_{i}\right)_{i \in \mathcal{C}}$ will be called a multi-strategy. The multi-strategies belong to the product strategy space $\mathcal{X}=\bigotimes_{i \in \mathcal{C}} \mathcal{X}_{i}$. 
Dispatcher $i$ seeks to find a routing strategy that minimizes the mean weighted sojourn times of its jobs, which, by Little's law, is equivalent to minimizing the mean weighted number of jobs in the system as seen by this class. This optimization problem, which depends on the routing decisions of the other classes, can be formulated as follows:

$$
\underset{\mathbf{x}_{i} \in \mathcal{X}_{i}}{\operatorname{minimize}} T_{i}(\mathbf{x})=\sum_{j \in \mathcal{S}} c_{j} \frac{x_{i, j}}{r_{j}-y_{j}}
$$

where $y_{j}=\sum_{k \in \mathcal{C}} x_{k, j}$ is the traffic offered to server $j$. Note that, introducing $r_{i, j}=r_{j}-\sum_{k \neq i} x_{k, j}$, the available capacity of server $j$ as seen by class $i$, the problem can alternatively be formulated as

$$
\underset{\mathbf{x}_{i} \in \mathcal{X}_{i}}{\operatorname{minimize}} \sum_{j \in \mathcal{S}} c_{j} \frac{x_{i, j}}{r_{i, j}-x_{i, j}} .
$$

A Nash equilibrium of the routing game is a multi-strategy from which no class finds it beneficial to deviate unilaterally. Hence, $\mathrm{x} \in \mathcal{X}$ is a Nash Equilibrium Point (NEP) if

$$
\mathbf{x}_{i}=\arg \min _{\mathbf{z} \in \mathcal{X}_{i}} T_{i}\left(\mathbf{x}_{1}, \ldots, \mathbf{x}_{i-1}, \mathbf{z}, \mathbf{x}_{i+1}, \ldots, \mathbf{x}_{K}\right), \quad \forall i \in \mathcal{C} .
$$

Let $T_{i j}(\mathbf{x})$ denote the partial derivative of $T_{i}$ with respect to $x_{i, j}$ at point $\mathbf{x}$, then

$$
T_{i j}(\mathbf{x})=c_{j}\left[\frac{1}{r_{j}-y_{j}}+\frac{x_{i, j}}{\left(r_{j}-y_{j}\right)^{2}}\right] .
$$

According to the Karush-Kuhn-Tucker (KKT) optimality conditions, $\mathbf{x} \in \mathcal{X}$ is a NEP if and only if there exist multipliers $\mu_{i}$ such that

$$
\begin{array}{r}
T_{i j}(\mathbf{x})=\frac{c_{j}}{r_{j}-y_{j}}+\frac{c_{j} x_{i, j}}{\left(r_{j}-y_{j}\right)^{2}}=\mu_{i} \quad \text { if } x_{i, j}>0 \\
T_{i j}(\mathbf{x})=\frac{c_{j}}{r_{j}-y_{j}} \geq \mu_{i} \quad \text { if } x_{i, j}=0 .
\end{array}
$$

Let $\mathcal{C}_{j}=\left\{i \in \mathcal{C}: x_{i, j}>0\right\}$ be the set of classes which route traffic to server $j$. Similarly, let $\mathcal{S}_{i}=\left\{j \in \mathcal{S}: x_{i, j}>0\right\}$ be the set of servers to which class $i$ routes traffic. Note that $i \in \mathcal{C}_{j} \Longleftrightarrow j \in \mathcal{S}_{i}$. We can now rewrite equations (3) and (4) as

$$
\frac{c_{j}}{r_{j}-y_{j}}<\mu_{i} \Longleftrightarrow i \in \mathcal{C}_{j} \Longleftrightarrow j \in \mathcal{S}_{i} .
$$

Let $\mathbf{x}$ be a NEP for the system with $K$ dispatchers. The global performance of the system can be assessed using the global cost

$$
D_{K}(\boldsymbol{\lambda}, \mathbf{r}, \mathbf{c})=\sum_{i \in \mathcal{C}} T_{i}(\mathbf{x})=\sum_{j \in \mathcal{S}} c_{j} \frac{y_{j}}{r_{j}-y_{j}},
$$


where the offered traffic $y_{j}$ are those at the NEP. The above cost represents the mean weighted number of jobs in the system. Note that when there is a single dispatcher, we have a single class whose traffic intensity is $\lambda_{1}=\bar{\lambda}$. The global cost can therefore be written as $D_{1}(\bar{\lambda}, \mathbf{r}, \mathbf{c})$ in this case.

We shall use the price of anarchy as a metric in order to assess the inefficiency of a decentralized scheme with $K$ dispatchers. For our problem, it is defined as

$$
\operatorname{PoA}(K)=\sup _{\boldsymbol{\lambda}, \mathbf{r}, \mathbf{c}} \frac{D_{K}(\boldsymbol{\lambda}, \mathbf{r}, \mathbf{c})}{D_{1}(\bar{\lambda}, \mathbf{r}, \mathbf{c})} .
$$

In the following section, we establish several important properties of the Nash equilibrium when the input parameters $\boldsymbol{\lambda}, \mathbf{r}$, and $\mathbf{c}$ are fixed. These properties will be used to prove the main results of this paper related to the PoA.

\subsection{Main Results}

Before getting into the technical details, we present here an overview of the most important results obtained in the paper.

The first theorem, which is proved in Section 5, states that that the global $\operatorname{cost} D_{K}(\boldsymbol{\lambda}, \mathbf{r}, \mathbf{c})$ achieves its maximum when $\boldsymbol{\lambda}$ is the symmetric vector $\boldsymbol{\lambda}^{=}=\left(\frac{\bar{\lambda}}{K}, \ldots, \frac{\bar{\lambda}}{K}\right)$.

\section{Theorem 1}

$$
\sup _{\boldsymbol{\lambda}, \mathbf{r}, \mathbf{c}} D_{K}(\boldsymbol{\lambda}, \mathbf{r}, \mathbf{c})=\sup _{\mathbf{r}, \mathbf{c}} D_{K}\left(\boldsymbol{\lambda}^{=}, \mathbf{r}, \mathbf{c}\right)
$$

This result implies that, for the calculation of the PoA, we can restrict ourselves to the symmetric game. This, coupled with the fact that in our setting the symmetric game is also a potential game, makes it more tractable for the analytic computation of the NEP and the global cost, thereby greatly simplifying the derivation of the lower and upper bounds on the PoA.

The second theorem, which is proved in Section 6, gives these lower and upper bounds on the PoA.

Theorem 2 For a system with two or more servers,

$$
\frac{K}{2 \sqrt{K}-1} \leq \operatorname{Po} A(K) \leq \sqrt{K} .
$$

This result states that the PoA is of the order of $\sqrt{K}$ independently of the number of servers, and thus remains bounded for a finite number of dispatchers.

Remark 1 For a system with only one server, $\operatorname{Po} A(K)=1$. Hence, we do not consider this case. 


\section{Existence, Uniqueness and Other Properties of The Nash Equilibrium}

In this section, we show the existence and uniqueness of the NEP and investigate properties of the traffic flow at this point.

\subsection{Existence and Uniqueness}

Let $\mathbf{x}$ be a multi-strategy for the routing game. The cost function $T_{i}(\mathbf{x})$ of each user $i \in \mathcal{C}$ is the sum over servers $j \in \mathcal{S}$ of the link cost functions $t_{i, j}(\mathbf{x})=$ $c_{j} x_{i, j} /\left(r_{j}-y_{j}\right)$. The latter are continuous functions of $\mathbf{x}$ which are convex and continuously differentiable in $x_{i, j}$. We have also assumed that $\bar{\lambda}<\bar{r}$. As stated in [18], these conditions are sufficient to guarantee the existence of a NEP (Theorem 1 in [19]).

Now observe that the function $t_{i, j}(\mathbf{x})$ is a function of two arguments, $x_{i, j}$ and $y_{j}$, which is increasing in each of its two arguments. Moreover, the partial derivative $T_{i, j}(\mathbf{x})$ is strictly increasing in $x_{i, j}$ and in $y_{j}$. We therefore conclude that the function $T_{i}(\mathbf{x})$ meets the conditions defining a type-A cost function in [18]. Hence, we can apply Theorem 2.1 in [18] and conclude that the NEP is unique.

\subsection{Properties related to traffic intensities}

We prove below that there is a monotonicity among classes in their use of servers: a class with a higher demand uses more of each and every server. We first prove a series of technical lemmata before stating our main results in Proposition 1 and Corollary 1.

Lemma $1 \mathcal{S}_{i} \cap \mathcal{S}_{k} \neq \emptyset$.

Proof. Assume the contrary, i.e., if $m \in \mathcal{S}_{i}$ then $m \notin \mathcal{S}_{k}$, and if $n \in \mathcal{S}_{k}$ then $n \notin \mathcal{S}_{i}$. For one such pair $m$ and $n$, from (5), we can conclude that $\mu_{i}>\frac{c_{m}}{r_{m}-y_{m}} \geq \mu_{k}$ and $\mu_{k}>\frac{c_{n}}{r_{n}-y_{n}} \geq \mu_{i}$, which is a contradiction.

Since $\mathcal{S}_{i} \cap \mathcal{S}_{k} \neq \emptyset$, from (3), we have

$$
\mu_{i}-\mu_{k}=\frac{c_{j}}{\left(r_{j}-y_{j}\right)^{2}}\left(x_{i, j}-x_{k, j}\right), \quad \forall j \in \mathcal{S}_{i} \cap \mathcal{S}_{k} .
$$

Lemma $2 \mu_{i}<\mu_{k} \Longleftrightarrow \exists j \in \mathcal{S}_{k}: x_{i, j}<x_{k, j}$.

Proof. Straight part: From Lemma $1, \mathcal{S}_{i} \cap \mathcal{S}_{k} \neq \emptyset$. If $\mu_{i}<\mu_{k}$, then, from (6), $\exists j \in \mathcal{S}_{k}: x_{i, j}<x_{k, j}$. 
Converse part: $\exists j \in \mathcal{S}_{k}: x_{i, j}<x_{k, j}$. Either $j \in \mathcal{S}_{i}$ or $j \notin \mathcal{S}_{i}$. If $j \in \mathcal{S}_{i}$ then, from (6), $\mu_{i}<\mu_{k}$. If $j \notin \mathcal{S}_{i}$, then, from (5), $\mu_{i} \leq \frac{c_{j}}{r_{j}-y_{j}}<\mu_{k}$.

Lemma 3 If $\mu_{i}<\mu_{k}$, then $\mathcal{S}_{i} \subset \mathcal{S}_{k}$.

Proof. If $j \in \mathcal{S}_{i}$, then, from (5), $\frac{c_{j}}{r_{j}-y_{j}}<\mu_{i}$. If $\mu_{i}<\mu_{k}$ then $\frac{c_{j}}{r_{j}-y_{j}}<\mu_{k}$. Hence, from (5) we can conclude that $j \in \mathcal{S}_{k}$. Therefore, $\mathcal{S}_{i} \subset \mathcal{S}_{k}$.

Lemma $4 \exists m \in \mathcal{S}_{k}: x_{i, m}<x_{k, m} \Longleftrightarrow x_{i, j}<x_{k, j}, \quad \forall j \in \mathcal{S}_{k}$.

Proof. Straight part: If $\exists m \in \mathcal{S}_{k}: x_{i, m}<x_{k, m}$, then, from Lemmata 2 and 3, we have $\mu_{i}<\mu_{k}$ and $\mathcal{S}_{i} \subset \mathcal{S}_{k}$. For $j \in \mathcal{S}_{i}$, from (6), we have $x_{i, j}<x_{k, j}$. For $j \in \mathcal{S}_{k} \backslash \mathcal{S}_{i}, x_{i, j}=0$ and $0<x_{k, j}$. Hence, $x_{i, j}<x_{k, j}, \forall j \in \mathcal{S}_{k}$.

Converse part: It is true from the statement.

Proposition 1 The following statements are equivalent:

1. $\mu_{i}<\mu_{k}$.

2. $\exists j \in \mathcal{S}_{k}: x_{i, j}<x_{k, j}$.

3. $x_{i, j}<x_{k, j}, \forall j \in \mathcal{S}_{k}$.

4. $\lambda_{i}<\lambda_{k}$.

Proof. $1 \Longleftrightarrow 2 \Longleftrightarrow 3$ follows from Lemmata 2 and 4 . Now, we show $3 \Longleftrightarrow 4$.

Straight part: If $x_{i, j}<x_{k, j}, \forall j \in \mathcal{S}_{k}$, then, from the fact that $3 \Longleftrightarrow 1$ and Lemma 3, we can conclude that $\lambda_{i}=\sum_{j \in \mathcal{S}_{i}} x_{i, j}=\sum_{j \in \mathcal{S}_{k}} x_{i, j}<\sum_{j \in \mathcal{S}_{k}} x_{k, j}=$ $\lambda_{k}$.

Converse part: Since $\lambda_{k}=\sum_{j \in \mathcal{S}_{k}} x_{k, j}$, if $\lambda_{i}<\lambda_{k}$, then $\exists j \in \mathcal{S}_{k}: x_{i, j}<x_{k, j}$. Since $2 \Longleftrightarrow 3$, if $\lambda_{i}<\lambda_{k}$, then $x_{i, j}<x_{k, j}, \forall j \in \mathcal{S}_{k}$.

The above proposition shows that a class with a higher demand uses more of each and every server. The following corollary shows that if two classes have the same traffic intensity, then they send the same amount of flow on each server.

Corollary 1 From Proposition 1, it follows that

1. $\mu_{i}=\mu_{k} \Longleftrightarrow \exists j \in \mathcal{S}_{k}: x_{i, j}=x_{k, j} \Longleftrightarrow x_{i, j}=x_{k, j}, \forall j \in \mathcal{S}_{k} \Longleftrightarrow \lambda_{i}=$ $\lambda_{k}$.

2. If $\lambda_{i}<\lambda_{k}$, then $\mathcal{S}_{i} \subset \mathcal{S}_{k}$. 
3. If $\lambda_{i}=\lambda_{k}$, then $\mathcal{S}_{i}=\mathcal{S}_{k}$.

In particular, if all classes have the same demand, i.e. $\boldsymbol{\lambda}=\boldsymbol{\lambda}^{=}$, then, for all server $j \in \mathcal{S}$ and for all $i \in \mathcal{C}$, we have $x_{i, j}=y_{j} / K$.

Recall that we have assumed that $\lambda_{i} \leq \lambda_{k}$ for $i \leq k$. Therefore, according to the above results, if we consider two classes $i$ and $k>i$, then we have $\mathcal{S}_{i} \subseteq \mathcal{S}_{k}$,

$\mu_{i} \leq \mu_{k}$ and $x_{i, j} \leq x_{k, j}$ for all servers $j \in \mathcal{S}_{k}$, with the equalities holding if and only if $\lambda_{i}=\lambda_{k}$.

Let $N_{j}=\left|\mathcal{C}_{j}\right|$. Proposition 1 implies that if $k \in \mathcal{C}_{j}$, then $i \in \mathcal{C}_{j}, \forall i>k$. As a consequence, the set $\mathcal{C}_{j}$ has the following structure: $\mathcal{C}_{j}=\left\{k-N_{j}+1, \ldots, K\right\}$.

\subsection{Properties related to server costs per unit capacity}

The above results tell how an order on $\lambda_{i}$ translates to an order on $x_{i, j}, \mu_{i}$ and $\mathcal{S}_{i}$, i.e., quantities of class $i$. We now give the analogous results for similar quantities of server $j$. As before, we first prove a series of technical lemmata before stating our main results in Proposition 2 and Corollary 2.

Denote $\frac{r_{j}-y_{j}}{c_{j}}=: \kappa_{j}$. We can rewrite (5) as

$$
\mu_{i}^{-1}<\kappa_{j} \Longleftrightarrow i \in \mathcal{C}_{j} \Longleftrightarrow j \in \mathcal{S}_{i} .
$$

Note that $\kappa_{m}$ is to class $m$ what $\mu_{i}$ is to class $i$. Also, for $i \in \mathcal{C}_{m}$, we can rewrite (3) as

$$
\begin{aligned}
\mu_{i} & =\frac{c_{m}}{r_{m}-y_{m}}\left(1+\frac{c_{m}}{r_{j}-y_{m}} \frac{x_{i, m}}{c_{m}}\right) \\
& =\kappa_{m}^{-1}\left(1+\kappa_{m}^{-1} \frac{x_{i, m}}{c_{m}}\right) .
\end{aligned}
$$

Lemma $5 \mathcal{C}_{m} \cap \mathcal{C}_{n} \neq \emptyset$

Proof. Assume the contrary, i.e., if $i \in \mathcal{C}_{m}$, then $i \notin \mathcal{C}_{n}$, and if $k \in \mathcal{C}_{n}$, then $k \notin \mathcal{C}_{m}$. For one such pair $i$ and $k$, from (7), we can conclude that $\kappa_{m}>\mu_{i}^{-1} \geq \kappa_{n}$ and $\kappa_{n}>\mu_{k}^{-1} \geq \kappa_{m}$, which is a contradiction.

Since $\mathcal{C}_{m} \cap \mathcal{C}_{n} \neq \emptyset$, from (8), we have

$$
\kappa_{m}^{-1}\left(1+\kappa_{m}^{-1} \frac{x_{i, m}}{c_{m}}\right)=\kappa_{n}^{-1}\left(1+\kappa_{n}^{-1} \frac{x_{i, n}}{c_{n}}\right), \quad \forall i \in \mathcal{C}_{m} \cap \mathcal{C}_{n}
$$

Lemma $6 \kappa_{m}<\kappa_{n} \Longleftrightarrow \exists i \in \mathcal{C}_{n}: \frac{x_{i, m}}{c_{m}}<\frac{x_{i, n}}{c_{n}}$. 
Proof. Straight part: From Lemma $5, \mathcal{C}_{m} \cap \mathcal{C}_{n} \neq \emptyset$. If $\kappa_{m}<\kappa_{n}$, then, from (9), $\exists i: \frac{x_{i, m}}{c_{m}}<\frac{x_{i, n}}{c_{n}}$.

Converse part: $\exists i \in \mathcal{C}_{n}: \frac{x_{i, m}}{c_{m}}<\frac{x_{i, n}}{c_{n}}$. Either $i \in \mathcal{C}_{m}$ or $i \notin \mathcal{C}_{m}$. If $i \in \mathcal{C}_{m}$, then, from (9), $\kappa_{m}<\kappa_{n}$. If $i \notin \mathcal{C}_{m}$, then, from (7), $\kappa_{m} \leq \mu_{i}^{-1}<\kappa_{n}$.

Lemma 7 If $\kappa_{m}<\kappa_{n}$, then $\mathcal{C}_{m} \subset \mathcal{C}_{n}$.

Proof. If $i \in \mathcal{C}_{m}$, then, from (7), $\mu_{i}<\kappa_{m}$. If $\kappa_{m}<\kappa_{n}$, then $\mu_{i}<\kappa_{n}$. Hence, from (7) we can conclude that $i \in \mathcal{C}_{n}$. Therefore, $\mathcal{C}_{m} \subset \mathcal{C}_{n}$.

Lemma $8 \exists m \in \mathcal{C}_{i}: \frac{x_{i, m}}{c_{m}}<\frac{x_{i, n}}{c_{n}} \Longleftrightarrow \frac{x_{i, m}}{c_{m}}<\frac{x_{i, n}}{c_{n}}, \forall i \in \mathcal{C}_{n}$.

Proof. Straight part: If $\exists i \in \mathcal{C}_{n}: \frac{x_{i, m}}{c_{m}}<\frac{x_{i, n}}{c_{n}}$, then, from Lemmata 6 and 7 , we have $\kappa_{m}<\kappa_{n}$ and $\mathcal{C}_{m} \subset \mathcal{C}_{n}$. For $i \in \mathcal{C}_{m}$, from (9), $\frac{x_{i, m}}{c_{m}}<\frac{x_{i, n}}{c_{n}}$. For $i \in \mathcal{C}_{n} \backslash \mathcal{C}_{m}$, $x_{i, m}=0$ and $0<x_{i, n}$. Hence, $\frac{x_{i, m}}{c_{m}}<\frac{x_{i, n}}{c_{n}}, \forall i \in \mathcal{C}_{n}$.

Converse part: It is true from the statement.

The following proposition proves a monotonic property regarding the order of preference of servers as seen by each class.

Proposition 2 The following statements are equivalent:

1. $\kappa_{m}<\kappa_{n}$.

2. $\exists i \in \mathcal{S}_{n}: \frac{x_{i, m}}{c_{m}}<\frac{x_{i, n}}{c_{n}}$.

3. $\frac{x_{i, m}}{c_{m}}<\frac{x_{i, n}}{c_{n}}, \forall i \in \mathcal{C}_{n}$.

4. $\frac{r_{m}}{c_{m}}<\frac{r_{n}}{c_{n}}$.

Proof. $1 \Longleftrightarrow 2 \Longleftrightarrow 3$ follows from Lemmata 6 and 8 . Next, we show $3 \Longleftrightarrow 4$.

Straight part: If $\frac{x_{i, m}}{c_{m}}<\frac{x_{i, n}}{c_{n}}, \forall i \in \mathcal{C}_{n}$, then from the fact that $3 \Longleftrightarrow 1$ and Lemma 7, we can conclude that $\frac{r_{m}}{c_{m}}=\kappa_{m}+\sum_{i \in \mathcal{C}_{m}} \frac{x_{i, m}}{c_{m}}<\kappa_{n}+\sum_{i \in \mathcal{C}_{n}} \frac{x_{i, n}}{c_{n}}=\frac{r_{n}}{c_{n}}$.

Converse part: Since $\frac{r_{n}}{c_{n}}=\kappa_{n}+\sum_{i \in \mathcal{C}_{n}} \frac{x_{i, n}}{c_{n}}$, if $\frac{r_{m}}{c_{m}}<\frac{r_{n}}{c_{n}}$, then either $\kappa_{m}<\kappa_{n}$ or $\exists i \in \mathcal{C}_{n}: \frac{x_{i, m}}{c_{m}}<\frac{x_{i, n}}{c_{n}}$. Since $1 \Longleftrightarrow 2 \Longleftrightarrow 3$, we can conclude that if $\frac{r_{m}}{c_{m}}<\frac{c_{n}}{r_{n}}$, then $\frac{x_{i, m}}{c_{m}}<\frac{x_{i, n}}{c_{n}}, \forall i \in \mathcal{C}_{n}$.

The above proposition shows that if server $n$ has a lower cost per unit capacity than server $m$, i.e. $c_{n} / r_{n}<c_{m} / r_{m}$, then, at the NEP, the ratio of the residual capacities will be greater than $c_{n} / c_{m}$, i.e. $\frac{r_{n}-y_{n}}{r_{m}-y_{m}}>\frac{c_{n}}{c_{m}}$, and for each class $i$ using server $n$, the ratio of the amount of traffic sent to each server by class $i$ will be greater than $c_{n} / c_{m}$, i.e. $\frac{x_{i, n}}{x_{i, m}}>\frac{c_{n}}{c_{m}}$. 
Corollary 2 From Proposition 2 it follows that

1. $\kappa_{m}=\kappa_{n} \Longleftrightarrow \exists i \in \mathcal{C}_{n}: \frac{x_{i, m}}{c_{m}}=\frac{x_{i, n}}{c_{n}} \Longleftrightarrow \frac{x_{i, m}}{c_{m}}=\frac{x_{i, n}}{c_{n}}, \forall i \in \mathcal{C}_{n} \Longleftrightarrow$ $\frac{r_{m}}{c_{m}}=\frac{r_{n}}{c_{n}}$.

2. If $\frac{r_{m}}{c_{m}}<\frac{r_{n}}{c_{n}}$ then $\mathcal{C}_{m} \subset \mathcal{C}_{n}$.

3. If $\frac{r_{m}}{c_{m}}=\frac{r_{n}}{c_{n}}$ then $\mathcal{C}_{m}=\mathcal{C}_{n}$.

The above corollary shows that we get a partition of classes among servers at the NEP: starting with a server $m$ of maximal cost per unit capacity $\frac{c_{m}}{r_{m}}$ and moving towards servers $n$ with lower cost per unit capacity $\frac{c_{n}}{r_{n}}<\frac{c_{m}}{r_{m}}$, we observe more and more classes joining the servers, i.e. $\mathcal{C}_{m} \subset \mathcal{C}_{n}$.

Recall that it is assumed that the servers are numbered in the following order: $c_{1} / r_{1} \leq c_{2} / r_{2} \leq \ldots \leq c_{S} / r_{S}$. According to the above properties, it implies that if we consider two servers $n$ and $m>n$, then we have $\mathcal{C}_{m} \subseteq \mathcal{C}_{n}$, $\frac{r_{n}-y_{n}}{r_{m}-y_{m}} \geq \frac{c_{n}}{c_{m}}$ and $\frac{x_{i, n}}{x_{i, m}} \geq \frac{c_{n}}{c_{m}}$ for each class $i \in \mathcal{C}_{n}$, with the equalities holding if and only if $c_{n} / r_{n}=c_{m} / r_{m}$.

Let $S_{i}=\left|\mathcal{S}_{i}\right|$. Proposition 2 implies that if $m \in \mathcal{S}_{i}$, then $n \in \mathcal{S}_{i}, \forall n<m$. As a consequence, the set $\mathcal{S}_{i}$ has the following structure: $\mathcal{S}_{i}=\left\{1, \ldots, S_{i}\right\}$.

Before moving to the analysis of the set of servers used by each class at the equilibrium, we conclude this section with a last property related to the server costs per unit capacity. This technical result will play a key role when comparing the costs of two different equilibria.

\section{Lemma 9}

$$
\frac{c_{j} r_{j}}{\left(r_{j}-y_{j}\right)^{2}} \geq \frac{c_{j+1} r_{j+1}}{\left(r_{j+1}-y_{j+1}\right)^{2}}, \forall j,
$$

with strict inequality if $\mathcal{C}_{j} \backslash \mathcal{C}_{j+1} \neq \emptyset$.

Proof. From (3), if $x_{i, j}>0$, then

$$
\mu_{i}=\frac{c_{j}}{r_{j}-y_{j}}+\frac{c_{j} x_{i, j}}{\left(r_{j}-y_{j}\right)^{2}},
$$

from which we conclude that

$$
\sum_{i \in \mathcal{C}_{j}} \mu_{i}=\frac{c_{j} N_{j}}{r_{j}-y_{j}}+\frac{c_{j} y_{j}}{\left(r_{j}-y_{j}\right)^{2}}=\frac{c_{j}\left(N_{j}-1\right)}{r_{j}-y_{j}}+\frac{c_{j} r_{j}}{\left(r_{j}-y_{j}\right)^{2}} .
$$


Now,

$$
\begin{aligned}
\frac{c_{j+1}\left(N_{j+1}-1\right)}{r_{j+1}-y_{j+1}}+\frac{c_{j+1} r_{j+1}}{\left(r_{j+1}-y_{j+1}\right)^{2}} & =\sum_{i \in \mathcal{C}_{j+1}} \mu_{i} \\
& =\sum_{i \in \mathcal{C}_{j}} \mu_{i}-\sum_{i \in \mathcal{C}_{j} \backslash \mathcal{C}_{j+1}} \mu_{i} \\
& =\frac{c_{j}\left(N_{j}-1\right)}{r_{j}-y_{j}}+\frac{c_{j} r_{j}}{\left(r_{j}-y_{j}\right)^{2}}-\sum_{i \in \mathcal{C}_{j} \backslash \mathcal{C}_{j+1}}\left(\frac{c_{j}}{r_{j}-y_{j}}+\frac{c_{j} x_{i, j}}{\left(r_{j}-y_{j}\right)^{2}}\right) \\
& =\frac{c_{j}\left(N_{j}-1\right)}{r_{j}-y_{j}}+\frac{c_{j} r_{j}}{\left(r_{j}-y_{j}\right)^{2}}-\frac{c_{j}\left(N_{j}-N_{j+1}\right)}{r_{j}-y_{j}}-\sum_{i \in \mathcal{C}_{j} \backslash \mathcal{C}_{j+1}} \frac{c_{j} x_{i, j}}{\left(r_{j}-y_{j}\right)^{2}} \\
& =\frac{c_{j}\left(N_{j+1}-1\right)}{r_{j}-y_{j}}+\frac{c_{j} r_{j}}{\left(r_{j}-y_{j}\right)^{2}}-\sum_{i \in \mathcal{C}_{j} \backslash \mathcal{C}_{j+1}} \frac{c_{j} x_{i, j}}{\left(r_{j}-y_{j}\right)^{2}} .
\end{aligned}
$$

Thus,

$$
\begin{aligned}
\frac{c_{j} r_{j}}{\left(r_{j}-y_{j}\right)^{2}}-\frac{c_{j+1} r_{j+1}}{\left(r_{j+1}-y_{j+1}\right)^{2}} & =\frac{c_{j+1}\left(N_{j+1}-1\right)}{r_{j+1}-y_{j+1}}-\frac{c_{j}\left(N_{j+1}-1\right)}{r_{j}-y_{j}}+\sum_{i \in \mathcal{C}_{j} \backslash \mathcal{C}_{j+1}} \frac{c_{j} x_{i, j}}{\left(r_{j}-y_{j}\right)^{2}} \\
& =\left(\frac{c_{j+1}}{r_{j+1}-y_{j+1}}-\frac{c_{j}}{r_{j}-y_{j}}\right)\left(N_{j+1}-1\right)+\sum_{i \in \mathcal{C}_{j} \backslash \mathcal{C}_{j+1}} \frac{c_{j} x_{i, j}}{\left(r_{j}-y_{j}\right)^{2}} .
\end{aligned}
$$

From Proposition 2, $\frac{c_{j}}{r_{j}} \leq \frac{c_{j+1}}{r_{j+1}}$ implies $\kappa_{j} \geq \kappa_{j+1}$, i.e. $\frac{c_{j+1}}{r_{j+1}-y_{j+1}} \geq \frac{c_{j}}{r_{j}-y_{j}}$. Since the second term on the RHS is strictly positive if $\mathcal{C}_{j} \backslash \mathcal{C}_{j+1} \neq \emptyset$, we can conclude that

$$
\frac{c_{j} r_{j}}{\left(r_{j}-y_{j}\right)^{2}}-\frac{c_{j+1} r_{j+1}}{\left(r_{j+1}-y_{j+1}\right)^{2}} \geq 0
$$

with strict inequality if $\mathcal{C}_{j} \backslash \mathcal{C}_{j+1} \neq \emptyset$.

\subsection{Characterization of the set of servers used}

The following proposition shows that the set of servers used by each class has the so-called water-filling structure. Recall that dispatcher $i$ solves the optimization problem (1).

Proposition 3 For each class $i \in \mathcal{C}$, the threshold $S_{i}$ is such that

$$
G_{i, S_{i}}<\lambda_{i} \leq G_{i, S_{i}+1}
$$

where 


$$
G_{i, s}=\sum_{j=1}^{s-1} r_{i, j}-\sqrt{\frac{r_{i, s}}{c_{s}}} \sum_{j=1}^{s-1} \sqrt{c_{j} r_{i, j}} \quad s=2, \ldots, S
$$

with $G_{i, 1}=0$ and $G_{i, S+1}=\sum_{j \in \mathcal{S}} r_{j}-\bar{\lambda}+\lambda_{i}$. Note that $G_{i, S+1}$ is the system capacity as seen by class $i$ jobs.

Proof. Let $A$ be a subset of $\mathcal{S}_{i}$. Since $r_{i, j}=r_{j}-y_{j}+x_{i, j}$, (3) can be rewritten as $\mu_{i}\left(r_{j}-y_{j}\right)^{2}=c_{j} r_{i, j}$ for all $j \in \mathcal{S}_{i}$. Summing over $j \in A$, we get

$$
\mu_{i}=\left(\frac{\sum_{j \in A} \sqrt{c_{j} r_{i, j}}}{\sum_{j \in A} r_{j}-y_{j}}\right)^{2} \quad A \subseteq \mathcal{S}_{i},
$$

which in the case $A=\mathcal{S}_{i}$ can be written as,

$$
\mu_{i}=\left(\frac{\sum_{j \in \mathcal{S}_{i}} \sqrt{c_{j} r_{i, j}}}{\sum_{j \in \mathcal{S}_{i}} r_{i, j}-\lambda_{i}}\right)^{2}
$$

since $\sum_{j \in \mathcal{S}_{i}} r_{j}-y_{j}=\sum_{j \in \mathcal{S}_{i}} r_{i, j}-x_{i, j}=\sum_{j \in \mathcal{S}_{i}} r_{i, j}-\lambda_{i}$.

Since $S_{i+1} \notin \mathcal{S}_{i}$, we have from (4),

$$
\mu_{i}=\left(\frac{\sum_{j \in \mathcal{S}_{i}} \sqrt{c_{j} r_{i, j}}}{\sum_{j \in \mathcal{S}_{i}} r_{i, j}-\lambda_{i}}\right)^{2} \leq \frac{c_{S_{i}+1}}{r_{S_{i}+1}-y_{S_{i}+1}},
$$

which yields

$$
\lambda_{i} \leq \sum_{j=1}^{S_{i}} r_{i, j}-\sqrt{\frac{r_{i, S_{i}+1}}{c_{S_{i}+1}}} \sum_{j=1}^{S_{i}} \sqrt{c_{j} r_{i, j}}=G_{i, S_{i}+1} .
$$

For the lower bound, observe that, since $S_{i} \in \mathcal{S}_{i}$, (3) holds for $j=S_{i}$. Therefore, using (14) with $A=\left\{1, \ldots, S_{i}-1\right\}$, we can write

$$
\mu_{i}=\left(\frac{\sum_{j=1}^{S_{i}-1} \sqrt{c_{j} r_{i, j}}}{\sum_{j=1}^{S_{i}-1} r_{j}-y_{j}}\right)^{2}=\frac{c_{S_{i}} r_{i, S_{i}}}{\left(r_{S_{i}}-y_{S_{i}}\right)^{2}}
$$

i.e., 


$$
\sum_{j=1}^{S_{i}-1} \sqrt{c_{j} r_{i, j}}=\frac{\sqrt{c_{S_{i}} r_{i, S_{i}}}}{r_{i, S_{i}}-x_{i, S_{i}}}\left(\sum_{j=1}^{S_{i}-1} r_{i, j}-x_{i, j}\right)>\sqrt{\frac{c_{S_{i}}}{r_{i, S_{i}}}}\left(\sum_{j=1}^{S_{i}-1} r_{i, j}-\lambda_{i}\right)
$$

from which we get

$$
G_{i, S_{i}}=\sum_{j=1}^{S_{i}-1} r_{i, j}-\sqrt{\frac{r_{i, S_{i}}}{c_{S_{i}}}} \sum_{j=1}^{S_{i}-1} \sqrt{c_{j} r_{i, j}}<\lambda_{i}
$$

Remark 2 In the special case $\lambda_{i}=G_{i, S_{i}+1}$, inequality (4) holds tight for $j=$ $S_{i}+1$. Therefore, in this case, we can define the set of servers used by class $i$ as $\mathcal{S}_{i}=\left\{1, \ldots, S_{i}, S_{i}+1\right\}$, where server $S_{i}+1$ is "marginally" used, with $x_{i, S_{i}+1}=0$.

From Corollary 1, we can conclude that the thresholds $S_{1}, \ldots, S_{K}$ satisfy the order $S_{1} \leq S_{2} \leq \ldots \leq S_{K}$.

\section{Analysis of the Global Cost}

In this section, it will be assumed that the capacity vector $\mathbf{r}$ and the cost vector c are fixed. Our goal is to prove that the global cost $D_{K}(\boldsymbol{\lambda}, \mathbf{r}, \mathbf{c})$ achieves its maximum in the symmetric case, i.e. when $\boldsymbol{\lambda}=\boldsymbol{\lambda}^{=}$.

For each rate vector $\boldsymbol{\lambda} \in \Lambda$, we already know that there exists a unique NEP $\mathrm{x} \in \mathcal{X}$. Let us define the function $\mathcal{N}: \Lambda \rightarrow \mathcal{X}$ such that for each vector $\boldsymbol{\lambda} \in \Lambda$, $\mathcal{N}(\boldsymbol{\lambda}) \in \mathcal{X}$ is this unique NEP. In the sequel, the function $\mathcal{N}$ will be called the Nash mapping. We have the following result.

Theorem 3 The Nash mapping $\mathcal{N}$ is a continuous function from $\Lambda$ into $\mathcal{X}$.

Proof. Note that for each vector $\boldsymbol{\lambda} \in \Lambda, \mathcal{N}(\boldsymbol{\lambda}) \in \mathcal{X} \subset \bigotimes_{i \in \mathcal{C}} \bigotimes_{j \in \mathcal{S}}\left[0, r_{j}\right]$ and the latter set is a compact set. As a consequence, in order to apply the closed graph theorem (see Appendix A), we only need to show that the graph $\mathcal{G}_{\mathcal{N}}$ of $\mathcal{N}$ is closed. Let us therefore consider a convergent sequence $\left(\lambda^{n}, x^{n}\right)_{n \in \mathbb{N}}$ of points in $\mathcal{G}_{\mathcal{N}}$, where $x^{n}=\mathcal{N}\left(\lambda^{n}\right)$. Let $(\lambda, x)$ denote the limit of this sequence. Note that $\lambda \in \Lambda$ since $\Lambda$ is closed as a topological space. We need to show that $x=\mathcal{N}(\lambda)$.

We first show that $\mathbf{x} \in \mathcal{X}$ and $\sum_{j \in \mathcal{S}} x_{i, j}=\lambda_{i}$ for each $i \in \mathcal{C}$. For $i \in \mathcal{C}$ and $j \in \mathcal{S}$ fixed, the sequence $\left(x_{i, j}^{n}\right)_{n \in \mathbb{N}}$ takes values in the closed set $\left[0, r_{j}\right]$ 
and converges to $x_{i, j}$, from which we deduce that $0 \leq x_{i, j} \leq r_{j}$ and thus that $\mathbf{x} \in \mathcal{X}$. Moreover, for each $n \in \mathbb{N}$ and each $i \in \mathcal{C}$, we have $\sum_{j \in \mathcal{S}} x_{i, j}^{n}=\lambda_{i}^{n}$. Since $x^{n} \rightarrow x$, we have for each class $i \in \mathcal{C}$,

$$
\lambda_{i}=\lim _{n \rightarrow \infty} \lambda_{i}^{n}=\lim _{n \rightarrow \infty} \sum_{j \in \mathcal{S}} x_{i, j}^{n}=\sum_{j \in \mathcal{S}} x_{i, j} .
$$

We therefore conclude that the limit point $\mathbf{x}$ is such that $\mathbf{x} \in \mathcal{X}$ and satisfies $\sum_{j \in \mathcal{S}} x_{i, j}=\lambda_{i}$ for each $i \in \mathcal{C}$. Let us now show that $\mathbf{x}$ is the unique NEP in $\mathcal{X}$ satisfying these conditions. For each $n \in \mathbb{N}$, since $\mathbf{x}^{n}$ is a NEP, there exist multipliers $\mu_{i}^{n}, i \in \mathcal{C}$, such that

$$
\begin{aligned}
\mu_{i}^{n} & =\frac{c_{j}}{r_{j}-y_{j}^{n}}+\frac{c_{j} x_{i, j}^{n}}{\left(r_{j}-y_{j}^{n}\right)^{2}} \text { if } x_{i, j}^{n}>0 \\
\mu_{i}^{n} & \leq \frac{c_{j}}{r_{j}-y_{j}^{n}} \text { if } x_{i, j}^{n}=0 .
\end{aligned}
$$

Now, let us show that there exist $\mu_{i}$ such that $\mu_{i}^{n} \rightarrow \mu_{i}$. To this end, let $y_{j}=\lim _{n \rightarrow \infty} y_{j}^{n}=\sum_{i \in \mathcal{C}} x_{i, j}$ for each $j \in \mathcal{S}$. Note that $y_{j}^{n}<r_{j}$ implies $y_{j} \leq r_{j}$. Let us first show that $y_{1}<r_{1}$. Assume by contradiction that $y_{1}=r_{1}$. Then we have

$$
\forall \epsilon>0, \exists N_{\epsilon}, \forall n \in \mathbb{N}, n \geq N_{\epsilon} \Longrightarrow r_{1}-y_{1}^{n}<\epsilon
$$

But, since $r_{j}-y_{j}^{n} \leq c_{j}\left(r_{1}-y_{1}^{n}\right) / c_{1}$ for all $j \in \mathcal{S}$, it implies that for each $\epsilon>0$, we can find $n$ sufficiently large such that $r_{j}-y_{j}^{n}<c_{j} \epsilon / c_{1}$ for all $j \in \mathcal{S}$, and thus,

$$
\sum_{j \in \mathcal{S}} r_{j}-\bar{\lambda}=\sum_{j \in \mathcal{S}} r_{j}-\sum_{j \in \mathcal{S}} y_{j}^{n}<\epsilon \sum_{j \in \mathcal{S}} \frac{c_{j}}{c_{1}}
$$

Since the above holds for each $\epsilon>0$, this is clearly a contradiction with our assumption $\bar{\lambda}<\sum_{j \in \mathcal{S}} r_{j}$. We therefore conclude that $y_{1}<r_{1}$. Now since each class uses server 1 , we have for each $n$,

$$
\mu_{i}^{n}=\frac{c_{1}}{r_{1}-y_{1}^{n}}+\frac{c_{1} x_{i, 1}^{n}}{\left(r_{1}-y_{1}^{n}\right)^{2}}
$$

Since $x^{n} \rightarrow x$ and $y_{1}^{n} \rightarrow y_{1}<r_{1}$, we conclude that the sequence $\left(\mu_{i}^{n}\right)_{n \in \mathbb{N}}$ convergences to a limit $\mu_{i}$ such that

$$
\mu_{i}=\frac{c_{1}}{r_{1}-y_{1}}+\frac{c_{1} x_{i, 1}}{\left(r_{1}-y_{1}\right)^{2}}
$$

Observe that it implies that $y_{j}<r_{j}$ for all $j \in \mathcal{S}$. Indeed, if we assume on the contrary that $y_{j}=\sum_{i \in \mathcal{C}} x_{i, j}=r_{j}$, then it implies that there exist $i \in \mathcal{C}$ such that $x_{i, j}=\epsilon>0$. But in turn it implies that for $n$ sufficiently large we have $x_{i, j}^{n}>\epsilon / 2$ and thus $\mu_{i}^{n}>\left(c_{j} \epsilon / 2\right) /\left(r_{j}-y_{j}^{n}\right)^{2}$. We then deduce from $y_{j}^{n} \rightarrow r_{j}$ that 
$\mu_{i}^{n} \rightarrow \infty$, which is a contradiction with $\mu_{i}^{n} \rightarrow \mu_{i}$. Therefore $y_{j}<r_{j}$ for all $j \in \mathcal{S}$.

Now, let us consider the complementary slackness conditions for each NEP $\mathbf{x}^{n}$, that is,

$$
x_{i, j}^{n}\left(\frac{c_{j}}{r_{j}-y_{j}^{n}}+\frac{c_{j} x_{i, j}^{n}}{\left(r_{j}-y_{j}^{n}\right)^{2}}-\mu_{i}^{n}\right)=0 .
$$

Taking the limit as $n \rightarrow \infty$, we obtain

$$
x_{i, j}\left(\frac{c_{j}}{r_{j}-y_{j}}+\frac{c_{j} x_{i, j}}{\left(r_{j}-y_{j}\right)^{2}}-\mu_{i}\right)=0 .
$$

Since the above are the necessary and sufficient optimality conditions for a point to be a NEP, we conclude that $x=\mathcal{N}(\lambda)$ and thus that the graph $\mathcal{G}_{\mathcal{N}}$ is closed. Applying the closed graph theorem yields the proof.

In order to prove that the global cost achieves its maximum in the symmetric case, we need to compare the equilibria $\mathcal{N}(\boldsymbol{\lambda})$ and $\mathcal{N}(\hat{\boldsymbol{\lambda}})$ that are induced by two different rate vectors $\boldsymbol{\lambda}$ and $\hat{\boldsymbol{\lambda}}$ in $\Lambda$. If the resulting equilibria are such that the set of servers over which each class sends its flow do not coincide at both equilibria, then the comparisons become extremely complex, if possible at all.

To avoid this difficulty, we proceed as follows. In section 5.1, we first prove some preliminary results concerning the comparison of the equilibria induced by two different rate vectors $\boldsymbol{\lambda}$ and $\hat{\boldsymbol{\lambda}}$, assuming that these equilibria are such that each class sends its flow to the same servers under both equilibria. In Section 5.2, we compare the equilibria induced by two different rate vectors $\boldsymbol{\lambda}$ and $\hat{\boldsymbol{\lambda}}$, assuming that (i) these equilibria are such that each class sends its flow to the same servers under both equilibria, and (ii) $\hat{\boldsymbol{\lambda}}$ is obtained from $\boldsymbol{\lambda}$ using a certain transformation. In Section 5.3 we exploit the continuity of the Nash mapping to show that the global cost increases under this transformation even when the set of servers is different at the two equilibria. Finally, in Section 5.4, we show that the symmetric rate vector $\boldsymbol{\lambda}^{=}$can be obtained from any rate vector $\boldsymbol{\lambda}$ with a finite number of such transformations.

\subsection{Preliminary Results}

In this section, we prove some lemmata that will be used in order to compare the Nash equilibria induced by two vectors $\boldsymbol{\lambda} \in \Lambda$ and $\hat{\boldsymbol{\lambda}} \in \Lambda$. In the sequel, if $z$ is a certain quantity related to the Nash equilibrium induced by the vector $\boldsymbol{\lambda}$ then we shall denote the corresponding quantity for vector $\hat{\boldsymbol{\lambda}}$ by $\hat{z}$.

Lemma 10 For $i \in \mathcal{C}_{j}$,

1. if $\hat{y}_{j}<y_{j}$ and $\hat{x}_{i, j} \leq x_{i, j}$, then $\hat{\mu}_{i}<\mu_{i}$. 
2. if $\hat{y}_{j} \leq y_{j}$ and $\hat{x}_{i, j} \leq x_{i, j}$, then $\hat{\mu}_{i} \leq \mu_{i}$.

3. if $\hat{y}_{j} \leq y_{j}$ and $\hat{x}_{i, j}<x_{i, j}$, then $\hat{\mu}_{i}<\mu_{i}$.

4. if $\hat{y}_{j}=y_{j}$ and $\hat{\mu}_{i}<\mu_{i}$, then $\hat{x}_{i, j}<x_{i, j}$.

Proof. Proof of part 1: for $i \in \mathcal{C}_{j}$, we rewrite (3) as

$$
x_{i, j}=\left(r_{j}-y_{j}\right)\left(\frac{r_{j}-y_{j}}{c_{j}} \mu_{i}-1\right) .
$$

Therefore, $\hat{x}_{i, j} \leq x_{i, j}$ is equivalent to

$$
\left(r_{j}-\hat{y}_{j}\right)\left(\frac{r_{j}-\hat{y}_{j}}{c_{j}} \hat{\mu}_{i}-1\right) \leq\left(r_{j}-y_{j}\right)\left(\frac{r_{j}-y_{j}}{c_{j}} \mu_{i}-1\right),
$$

which, since $r_{j}-y_{j}<r_{j}-\hat{y}_{j}$, is equivalent to

$$
\left(r_{j}-\hat{y}_{j}\right)\left(\frac{r_{j}-\hat{y}_{j}}{c_{j}} \hat{\mu}_{i}-1\right)<\left(r_{j}-\hat{y}_{j}\right)\left(\frac{r_{j}-y_{j}}{c_{j}} \mu_{i}-1\right),
$$

which is equivalent to

$$
\frac{r_{j}-\hat{y}_{j}}{c_{j}} \hat{\mu}_{i}<\frac{r_{j}-y_{j}}{c_{j}} \mu_{i} .
$$

Since $r_{j}-y_{j}<r_{j}-\hat{y}_{j}$, we can conclude that $\hat{\mu}_{i}<\mu_{i}$.

The proofs of parts 2,3 , and 4 follow similarly.

Lemma 11 For $m$ and $n$ in $\mathcal{S}$, and $i \in \mathcal{C}_{m} \cap \mathcal{C}_{n}$,

if $\hat{y}_{m}>y_{m}, \hat{x}_{i, m} \geq x_{i, m}$, and $\hat{y}_{n} \leq y_{n}$, then $\hat{x}_{i, n}>x_{i, n}$.

Proof. Assume the contrary, that is, $\exists n, m \in \mathcal{S}$ and $i \in \mathcal{C}_{m} \cap \mathcal{C}_{n}$ such that $\hat{y}_{m}>y_{m}, \hat{x}_{i, m} \geq x_{i, m}, \hat{y}_{n} \leq y_{n}$ and $\hat{x}_{i, n} \leq x_{i, n}$. From Lemma 10.1, $\hat{y}_{m}>y_{m}$ and $\hat{x}_{i, m} \geq x_{i, m}$ implies $\hat{\mu}_{i}>\mu_{i}$. However, from Lemma 10.2, $\hat{y}_{n} \leq y_{n}$ and $\hat{x}_{i, n} \leq x_{i, n}$ implies $\hat{\mu}_{i} \leq \mu_{i}$, which is a contradiction.

In the rest of the section, we shall make the following assumption on the vectors $\boldsymbol{\lambda}$ and $\hat{\boldsymbol{\lambda}}$.

Assumption 1 The vectors $\boldsymbol{\lambda}$ and $\hat{\boldsymbol{\lambda}}$ are such that $\mathcal{C}_{j}=\hat{\mathcal{C}}_{j}, \forall j \in \mathcal{S}$.

From the above assumption, it follows that $\mathcal{S}_{i}=\hat{\mathcal{S}}_{i}, \forall i \in \mathcal{C}$.

Lemma 12 For any $j \in \mathcal{S}$ : 
1. $\hat{y}_{j} \geq y_{j} \Longleftrightarrow \sum_{i \in \mathcal{C}_{j}} \hat{\mu}_{i} \geq \sum_{i \in \mathcal{C}_{j}} \mu_{i}$.

2. $\hat{y}_{j}>y_{j} \Longleftrightarrow \sum_{i \in \mathcal{C}_{j}} \hat{\mu}_{i}>\sum_{i \in \mathcal{C}_{j}} \mu_{i}$.

Proof. Proof of part 1: from (2) and (3), if $i \in \mathcal{C}_{j}$, then

$$
\mu_{i}=c_{j}\left[\frac{1}{r_{j}-y_{j}}+\frac{x_{i, j}}{\left(r_{j}-y_{j}\right)^{2}}\right] .
$$

Thus,

$$
\sum_{i \in \mathcal{C}_{j}} \mu_{i}=n_{j} c_{j} \frac{1}{r_{j}-y_{j}}+c_{j} \frac{y_{j}}{\left(r_{j}-y_{j}\right)^{2}} .
$$

Since $N_{j}=\hat{N}_{j}$ (from Assumption 1), we can conclude that $\sum_{i \in \mathcal{C}_{j}} \mu_{i}$ is an increasing function of $y_{j}$.

The proof of part 2 follows similarly.

Lemma 13 If $\mathcal{C}_{m}=\mathcal{C}_{n}$ then :

1. $\hat{y}_{m} \geq y_{m} \Longleftrightarrow \hat{y}_{n} \geq y_{n}$.

2. $\hat{y}_{m}>y_{m} \Longleftrightarrow \hat{y}_{n}>y_{n}$.

Proof. Proof of part 1: from Lemma 12, $\hat{y}_{m} \geq y_{m}$ is equivalent to $\sum_{i \in \mathcal{S}_{m}} \hat{\mu}_{i} \geq$ $\sum_{i \in \mathcal{S}_{m}} \mu_{i}$, which, since $\mathcal{C}_{m}=\mathcal{C}_{n}$, is equivalent to $\sum_{i \in \mathcal{S}_{n}} \hat{\mu}_{i} \geq \sum_{i \in \mathcal{S}_{n}} \mu_{i}$. Again, from Lemma 12, we can conclude that $\hat{y}_{n} \geq y_{n}$.

The proof of part 2 follows similarly.

Corollary 3 For $m, n \in \mathcal{S}_{1}, \hat{y}_{m}>y_{m} \Longleftrightarrow \hat{y}_{n}>y_{n}$.

Proof. Since, for $m, n \in \mathcal{S}_{1}, \mathcal{C}_{m}=\mathcal{C}_{n}=\{1,2, \ldots, K\}$, the above statement follows from Lemma 13.2.

\subsection{Basic Transformation of a Rate Vector}

For each rate vector $\lambda \in \Lambda$, recall that by convention $\lambda_{1}=\min _{i \in \mathcal{C}} \lambda_{i}$ and $\lambda_{K}=\max _{i \in \mathcal{C}} \lambda_{i}$. Define the sets $\mathcal{C}_{\min }$ and $\mathcal{C}_{\max }$ as follows:

$$
\begin{aligned}
\mathcal{C}_{\text {min }} & =\left\{i \in \mathcal{C}: \lambda_{i}=\lambda_{1}\right\}, \\
\mathcal{C}_{\text {max }} & =\left\{i \in \mathcal{C}: \lambda_{i}=\lambda_{K}\right\},
\end{aligned}
$$

and let $n_{\text {min }}=\left|\mathcal{C}_{\text {min }}\right|$ and $n_{\text {max }}=\left|\mathcal{C}_{\text {max }}\right|$. 
Definition 1 For each rate vector $\boldsymbol{\lambda} \in \Lambda$, define the function $h_{\boldsymbol{\lambda}}:\left[0, n_{\max } \lambda_{K}\right] \rightarrow$ $\Lambda$ as follows:

$$
h_{\boldsymbol{\lambda}}(\epsilon)=\boldsymbol{\lambda}+\epsilon\left(\frac{1}{n_{\min }} \sum_{i \in \mathcal{C}_{\min }} \mathbf{e}_{i}-\frac{1}{n_{\max }} \sum_{i \in \mathcal{C}_{\max }} \mathbf{e}_{i}\right),
$$

where $\mathbf{e}_{i}$ denotes the vector in $\mathbb{R}^{K}$ with the $i$-th component equals to 1 and all other components are equal to 0 . A rate vector $\hat{\boldsymbol{\lambda}} \in \Lambda$ is said to be obtained from $\boldsymbol{\lambda}$ under a basic transformation if and only if there exists $\epsilon \in\left[0, n_{\max } \lambda_{K}\right]$ such that $\hat{\boldsymbol{\lambda}}=h_{\boldsymbol{\lambda}}(\epsilon)$. In this case, $\epsilon$ is called the step of the transformation.

Note that the above transformation increases the traffic of classes $i \in \mathcal{C}_{\text {min }}$ (the classes with the smallest amount of traffic) and decrease correspondingly the traffic of classes $i \in \mathcal{C}_{\max }$ (the classes with the largest amount of traffic), i.e., it preserves the total amount of traffic: $\sum_{i \in \mathcal{C}} \lambda_{i}=\sum_{i \in \mathcal{C}} \hat{\lambda}_{i}=\bar{\lambda}$. There are several other properties of the basic transformation which are worthwhile noticing. They are stated in the following lemma.

Lemma 14 Let $\hat{\boldsymbol{\lambda}}$ be obtained from $\boldsymbol{\lambda}$ under a basic transformation, i.e., $\hat{\boldsymbol{\lambda}}=$ $h_{\boldsymbol{\lambda}}(\epsilon)$. Then,

1. $\hat{\lambda}_{i} \geq \lambda_{i}$ for all $i \in \mathcal{C}_{\text {min }}, \hat{\lambda}_{i} \leq \lambda_{i}$ for all $i \in \mathcal{C}_{\text {max }}$, and $\hat{\lambda}_{i}=\lambda_{i}$ for all $i \notin \mathcal{C}_{\text {min }} \cup \mathcal{C}_{\text {max }}$, where the inequalities are strict if and only if $\mathcal{C}_{\text {min }} \neq \mathcal{C}_{\text {max }}$ and $\epsilon>0$.

2. $\hat{\lambda}_{i}=\hat{\lambda}_{1}$ for all $i \in \mathcal{C}_{\text {min }}$ and $\hat{\lambda}_{i}=\hat{\lambda}_{K}$ for all $i \in \mathcal{C}_{\text {max }}$,

3. $\hat{\lambda}_{i} \leq \lambda_{K}$ for all $i \in \mathcal{C}_{\text {min }}$ and $\hat{\lambda}_{i} \geq \lambda_{1}$ for all $i \in \mathcal{C}_{\text {max }}$ for $\epsilon \leq \min \left(n_{\min }, n_{\max }\right)\left(\lambda_{K}-\right.$ $\left.\lambda_{1}\right)$.

4. $\sum_{i \in \mathcal{C}_{\text {min }}} \hat{\lambda}_{i}-\lambda_{i}=-\sum_{i \in \mathcal{C}_{\max }} \hat{\lambda}_{i}-\lambda_{i}$,

5. $\sum_{j \in \mathcal{S}} y_{j}=\sum_{j \in \mathcal{S}} \hat{y}_{j}$,

Proof. In part 1 , if either $\mathcal{C}_{\min }=\mathcal{C}_{\max }$ or $\epsilon=0$, then $\hat{\boldsymbol{\lambda}}=\boldsymbol{\lambda}$. Hence, the equalities are satisfied. So, we consider the case when $\mathcal{C}_{\text {min }} \neq \mathcal{C}_{\text {max }}$ and $\epsilon>0$, and show that the inequalities are strict. From (18), it is immediate that $\hat{\lambda}_{i}=\lambda_{i}$ for $i \notin \mathcal{C}_{\min } \cup \mathcal{C}_{\max }$. Moreover $\hat{\lambda}_{i}=\lambda_{i}+\frac{\epsilon}{n_{\min }}$ for all $i \in \mathcal{C}_{\min }$ and $\hat{\lambda}_{i}=\lambda_{i}-\frac{\epsilon}{n_{\max }}$ for all $i \in \mathcal{C}_{\text {max }}$. Since $\epsilon>0$, we thus obtain $\hat{\lambda}_{i}>\lambda_{i}$ for $i \in \mathcal{C}_{\text {min }}$ and $\hat{\lambda}_{i}<\lambda_{i}$ for $i \in \mathcal{C}_{\max }$, and 1 is proved.

We note that if $\mathcal{C}_{\text {min }}=\mathcal{C}_{\text {max }}$, then $\hat{\boldsymbol{\lambda}}=\boldsymbol{\lambda}$. Hence, the parts 2 to 5 are true in this case. So, for the rest of the proof, we assume that $\mathcal{C}_{\text {min }} \neq \mathcal{C}_{\text {max }}$. From $\lambda_{i}=\lambda_{1}$ for $i \in \mathcal{C}_{\text {min }}$ and $\lambda_{i}=\lambda_{K}$ for $i \in \mathcal{C}_{\text {max }}$, we obtain that $\hat{\lambda}_{i}=\lambda_{1}+\frac{\epsilon}{n_{\min }}=$ $\hat{\lambda}_{1}$ for $i \in \mathcal{C}_{\text {min }}$ and $\hat{\lambda}_{i}=\lambda_{K}+\frac{\epsilon}{n_{\max }}=\hat{\lambda}_{K}$ for $i \in \mathcal{C}_{\text {max }}$, which proves 2 . 
To prove 3 , let us consider $i \in \mathcal{C}_{\text {min }}$. We observe that $\epsilon \leq \min \left(n_{\min }, n_{\max }\right)\left(\lambda_{K}-\right.$ $\left.\lambda_{1}\right)$ implies that $\hat{\lambda}_{i}=\lambda_{i}+\frac{\epsilon}{n_{\min }} \leq \lambda_{i}+\frac{\min \left(n_{\min }, n_{\max }\right)}{n_{\min }}\left(\lambda_{K}-\lambda_{1}\right) \leq \lambda_{i}+\left(\lambda_{K}-\lambda_{1}\right)=$ $\lambda_{K}$. The proof of $\hat{\lambda}_{i} \geq \lambda_{1}$ for $i \in \mathcal{C}_{\text {max }}$ is symmetric.

To prove 4 , we observe that,

$$
\sum_{i \in \mathcal{C}_{\min }} \hat{\lambda}_{i}-\lambda_{i}=\sum_{i \in \mathcal{C}_{\min }} \frac{\epsilon}{n_{\min }}=\epsilon=\sum_{i \in \mathcal{C}_{\max }} \frac{\epsilon}{n_{\max }}=-\sum_{i \in \mathcal{C}_{\max }} \hat{\lambda}_{i}-\lambda_{i}
$$

Finally, the proof of property 5 is immediate since it is equivalent to $\sum_{i \in \mathcal{C}} \lambda_{i}=$ $\sum_{i \in \mathcal{C}} \hat{\lambda}_{i}$

In the following, we will compare two rate vectors $\boldsymbol{\lambda}$ and $\hat{\boldsymbol{\lambda}}$. If $z$ is a certain quantity related to the Nash equilibrium induced by the vector $\boldsymbol{\lambda}$ then we shall denote the corresponding quantity for vector $\hat{\lambda}$ by $\hat{z}$. The comparison is done under the following assumption.

Assumption 2 The rate vectors $\boldsymbol{\lambda} \in \Lambda$ and $\hat{\boldsymbol{\lambda}} \in \Lambda$ are such that:

1. $\hat{\boldsymbol{\lambda}}$ is obtained from $\boldsymbol{\lambda}$ under a basic transformation,

2. $\mathcal{C}_{j}=\hat{\mathcal{C}}_{j}, \forall j \in \mathcal{S}$.

In other words, we assume that the transformation $\boldsymbol{\lambda}$ into $\hat{\boldsymbol{\lambda}}$ leaves unaffected the set of servers used by each class.

The key point here is that in order to determine the impact of a basic transformation of the rate vector $\boldsymbol{\lambda}$ on the global cost, we need to compare the server loads under the equilibria $\mathbf{x}=\mathcal{N}(\boldsymbol{\lambda})$ and $\hat{\mathbf{x}}=\mathcal{N}(\hat{\boldsymbol{\lambda}})$. To this end, let us define the sets $\mathcal{S}^{+}$and $\mathcal{S}^{-}$as follows:

$$
\mathcal{S}^{+}=\left\{j \in \mathcal{S}: \hat{y}_{j}>y_{j}\right\} \quad \text { and } \quad \mathcal{S}^{-}=\mathcal{S} \backslash \mathcal{S}^{+},
$$

i.e., $\mathcal{S}^{+}$is the set of servers whose load increases under the transformation while $\mathcal{S}^{-}$is the set of servers whose load is non-increasing under the transformation.

We first prove two lemmata concerning the sets $\mathcal{S}^{+}$and $\mathcal{S}^{-}$. The first one shows that $\mathcal{S}^{+}$is empty if and only if the load of each and every server is constant under the transformation.

Lemma $15 y_{j}=\hat{y}_{j}, \forall j \in \mathcal{S} \Longleftrightarrow \mathcal{S}^{+}=\emptyset$.

Proof. If $\mathcal{S}^{+}=\emptyset$ then $\mathcal{S}^{-}=\mathcal{S}$. That is, $\hat{y}_{j} \leq y_{j}, \forall j \in \mathcal{S}$. We also have $\sum_{j \in \mathcal{S}} \hat{y}_{j}=\sum_{j \in \mathcal{S}} y_{j}$. This is possible only if $\hat{y}_{j}=y_{j}, \forall j \in \mathcal{S}$.

The converse is true by definition of $\mathcal{S}^{+}$.

The second lemma shows that $\mathcal{S}^{-}$cannot be empty, i.e. that there is at least one server whose load is non-increasing under the transformation. 
Lemma $16 \mathcal{S}^{-} \neq \emptyset$.

Proof. Assume $\mathcal{S}^{-}=\emptyset$, then $y_{j}<\hat{y}_{j}, \forall j \in \mathcal{S}$. Therefore, $\sum_{j \in \mathcal{S}} y_{j}<\sum_{j \in \mathcal{S}} \hat{y}_{j}$. This is in contradiction with Assumption 2 which says $\sum_{j \in \mathcal{S}} y_{j}=\sum_{j \in \mathcal{S}} \hat{y}_{j}$.

We now prove three fundamental propositions regarding the impact of the transformation on server loads. We first show in proposition 4 that if there exists at least one server whose load increases under the transformation, then the load of each and every server used by class 1 increases. We then prove in proposition 5 that the load of all servers is non-increasing under the transformation if and only if all traffic classes use the same set of servers. Finally, proposition 6 proves that the transformation induces a monotonic partition of servers: there exists a threshold $J<S$ such that for all servers $j>J$ the load is non-increasing under the transformation.

Proposition 4 If $\mathcal{S}^{+} \neq \emptyset$ then $\mathcal{S}_{1} \subset \mathcal{S}^{+}$.

Proof. Assume by contradiction that we can find a server $s \in \mathcal{S}_{1}$ such that $s \in \mathcal{S}^{-}$. Then, according to Corollary $3, \mathcal{S}_{1} \subset \mathcal{S}^{-}$. Since $\mathcal{S}^{+} \neq \emptyset$ and $\hat{y}_{j}>y_{j}$ for all $j \in \mathcal{S}^{+}$, we have $\sum_{j \in \mathcal{S}^{+}} \hat{y}_{j}>\sum_{j \in \mathcal{S}^{+}} y_{j}$, i.e.,

$$
\sum_{i \in \mathcal{C}}\left(\sum_{j \in \mathcal{S}^{+}} \hat{x}_{i, j}\right)>\sum_{i \in \mathcal{C}}\left(\sum_{j \in \mathcal{S}^{+}} x_{i, j}\right)
$$

from which we conclude that there exists $i$ such that $\sum_{j \in \mathcal{S}^{+}} \hat{x}_{i, j}>\sum_{j \in \mathcal{S}^{+}} x_{i, j}$. Since $\mathcal{S}_{k}=\mathcal{S}_{1} \subset \mathcal{S}^{-}$for all $k \in \mathcal{C}_{\text {min }}$, we necessarily have $i \notin \mathcal{C}_{\text {min }}$ and thus $\hat{\lambda}_{i} \leq \lambda_{i}$. Therefore,

$$
\hat{\lambda}_{i}=\sum_{j \in \mathcal{S}^{-}} \hat{x}_{i, j}+\sum_{j \in \mathcal{S}^{+}} \hat{x}_{i, j} \leq \sum_{j \in \mathcal{S}^{-}} x_{i, j}+\sum_{j \in \mathcal{S}^{+}} x_{i, j}=\lambda_{i} .
$$

Thus,

$$
\sum_{j \in \mathcal{S}^{-}} \hat{x}_{i, j} \leq \sum_{j \in \mathcal{S}^{-}} x_{i, j}+\left(\sum_{j \in \mathcal{S}^{+}} x_{i, j}-\sum_{j \in \mathcal{S}^{+}} \hat{x}_{i, j}\right)<\sum_{j \in \mathcal{S}^{-}} x_{i, j}
$$

We therefore conclude that class $i$ is such that $\sum_{j \in \mathcal{S}^{+}} \hat{x}_{i, j}>\sum_{j \in \mathcal{S}^{+}} x_{i, j}$ and $\sum_{j \in \mathcal{S}^{-}} \hat{x}_{i, j}<\sum_{j \in \mathcal{S}^{-}} x_{i, j}$. Therefore, we can find a server $m \in \mathcal{S}^{+}$and a server $n \in \mathcal{S}^{-}$such that $\hat{x}_{i, m}>x_{i, m}$ and $\hat{x}_{i, n}<x_{i, n}$. But according to Lemma 11, this is impossible. We therefore conclude that $\mathcal{S}_{1} \subset \mathcal{S}^{+}$.

Proposition $5 \mathcal{S}^{+}=\emptyset \Longleftrightarrow \mathcal{S}_{1}=\mathcal{S}_{K}$. 
Proof. We first prove that if $S^{+}=\emptyset$ then $\mathcal{S}_{1}=\mathcal{S}_{K}$. From Lemma 15, this is equivalent to proving that if $y_{j}=\hat{y}_{j}, \forall j \in \mathcal{S}$ then $\mathcal{S}_{1}=\mathcal{S}_{K}$. Assume the contrary, that is $\mathcal{S}_{1} \subsetneq \mathcal{S}_{K}$. Then, $\exists m: m \in S_{K}, m \notin S_{1}$.

Since $y_{m}=\hat{y}_{m}$, from Lemma 12 , we get $\sum_{i \in \mathcal{C}_{m}} \mu_{i}=\sum_{i \in \mathcal{C}_{m}} \hat{\mu}_{i}$, which we can rewrite as

$$
\sum_{i \in \mathcal{C}_{\max }} \mu_{i}+\sum_{i \in \mathcal{C}_{m} \backslash \mathcal{C}_{\max }} \mu_{i}=\sum_{i \in \mathcal{C}_{\max }} \hat{\mu}_{i}+\sum_{i \in \mathcal{C}_{m} \backslash \mathcal{C}_{\max }} \hat{\mu}_{i}
$$

We shall show that the above equality is not possible, which then proves the claim.

For $i \in \mathcal{C}_{\text {max }}$, since $\lambda_{i}>\hat{\lambda}_{i}, \sum_{j \in \mathcal{S}_{i}} x_{i, j}>\sum_{j \in \mathcal{S}_{i}} \hat{x}_{i, j}$. Thus, there exists an $n \in \mathcal{S}_{i}$ such that $x_{i, n}>\hat{x}_{i, n}$. Since $y_{n}=\hat{y}_{n}$, from Lemma 10.3, we can conclude that $\mu_{i}>\hat{\mu}_{i}$, and that $\sum_{i \in \mathcal{C}_{m a x}} \mu_{i}>\sum_{i \in \mathcal{C}_{\text {min }}} \hat{\mu}_{i}$, which, upon substitution in (19), leads to

$$
\sum_{i \in \mathcal{C}_{m} \backslash \mathcal{C}_{\max }} \mu_{i}<\sum_{i \in \mathcal{C}_{m} \backslash \mathcal{C}_{\max }} \hat{\mu}_{i}
$$

If $\mathcal{C}_{m} \backslash \mathcal{C}_{\max }=\emptyset$, then the above inequality cannot be possible which then proves the claim. So, assume $\mathcal{C}_{m} \backslash \mathcal{C}_{\max } \neq \emptyset$. Then the above inequality implies that $\exists i \notin C_{\min } \cup C_{\max }: \mu_{i}<\hat{\mu}_{i}$. Since $y_{j}=\hat{y}_{j}, \forall j \in \mathcal{S}_{i}$, application of Lemma 10.4 leads to $x_{i, j}<\hat{x}_{i, j}, \forall j \in \mathcal{S}_{i}$, and consequently to $\lambda_{i}=\sum_{j \in \mathcal{S}_{i}} x_{i, j}<\sum_{j \in \mathcal{S}_{i}} \hat{x}_{i, j}=$ $\hat{\lambda}_{i}$. However, for $i \notin \mathcal{C}_{\min } \cup \mathcal{C}_{\max }$, from Lemma 14.1, $\lambda_{i}=\hat{\lambda}_{i}$. Hence, there is a contradiction, and we can conclude that $\mathcal{S}_{1}=\mathcal{S}_{K}$.

As a direct consequence of the above proposition, we get the following corollary that tells us that if at equilibria $\mathbf{x}$ and $\hat{\mathbf{x}}$ all classes use the same set of servers, then the server loads are constant under the transformation.

Corollary $4 y_{j}=\hat{y}_{j}, \forall j \in \mathcal{S} \Longleftrightarrow \mathcal{S}_{1}=\mathcal{S}_{K}$.

We now turn our attention to the set $\mathcal{S}^{-}$and prove the following result.

Proposition 6 For all $j \in \mathcal{S}$, if $j \in \mathcal{S}^{-}$then $j+1 \in \mathcal{S}^{-}$.

Proof. If $\mathcal{S}^{+}=\emptyset$ then the proposition is true. So, assume $\mathcal{S}^{+} \neq \emptyset$. Then, from Proposition $4, \mathcal{S}_{1} \subset \mathcal{S}^{+}$. In order to prove the proposition, assume by contradiction that there exists a server $j \in\left\{S_{1}+1, \ldots, S_{K}-1\right\}$ such that $j \in \mathcal{S}^{-}$and $j+1 \in \mathcal{S}^{+}$. Again, if $S_{1}+1=S_{K}$ then the proposition is true. So, assume that $S_{1}+1<S_{K}$.

Since $j \in \mathcal{S}^{-}$and $j+1 \in \mathcal{S}^{+}$, from Lemma 13 ,

$$
\sum_{i \in \mathcal{C}_{j}} \hat{\mu}_{i} \leq \sum_{i \in \mathcal{C}_{j}} \mu_{i},
$$


and

$$
\sum_{i \in \mathcal{C}_{j+1}} \hat{\mu}_{i}>\sum_{i \in \mathcal{C}_{j+1}} \mu_{i}
$$

Moreover, from the contrapositive of Lemma 13, we can conclude that $\mathcal{C}_{j} \backslash \mathcal{C}_{j+1} \neq$ $\emptyset$. Note that since $j<S_{K}$, classes $i \in \mathcal{C}_{\max }$ do not belong to $\mathcal{C}_{j} \backslash \mathcal{C}_{j+1}$. Similarly, since $j>S_{1}$, classes $i \in \mathcal{C}_{\text {min }}$ do not belong to $\mathcal{C}_{j} \backslash \mathcal{C}_{j+1}$.

Since $\mathcal{C}_{j+1} \subset \mathcal{C}_{j}$, we have, for all $i \in \mathcal{C}_{j+1}$,

$$
\mu_{i}=\frac{c_{j}}{r_{j}-y_{j}}+c_{j} \frac{x_{i, j}}{\left(r_{j}-y_{j}\right)^{2}} .
$$

Therefore, $\sum_{i \in \mathcal{C}_{j+1}} \hat{\mu}_{i}>\sum_{i \in \mathcal{C}_{j+1}} \mu_{i}$ is equivalent to

$$
N_{j+1} \frac{c_{j}}{r_{j}-\hat{y}_{j}}+\frac{c_{j}}{\left(r_{j}-\hat{y}_{j}\right)^{2}} \sum_{i \in \mathcal{C}_{j+1}} \hat{x}_{i, j}>N_{j+1} \frac{c_{j}}{r_{j}-y_{j}}+\frac{c_{j}}{\left(r_{j}-y_{j}\right)^{2}} \sum_{i \in \mathcal{C}_{j+1}} x_{i, j}
$$

and since $\hat{y}_{j} \leq y_{j}$, this implies that $\sum_{i \in \mathcal{C}_{j+1}} \hat{x}_{i, j}>\sum_{i \in \mathcal{C}_{j+1}} x_{i, j}$. Since $\hat{y}_{j} \leq y_{j}$, necessarily $\sum_{i \in \mathcal{C}_{j} \backslash \mathcal{C}_{j+1}} \hat{x}_{i, j}<\sum_{i \in \mathcal{C}_{j} \backslash \mathcal{C}_{j+1}} x_{i, j}$. However, since all classes $k \in$ $\mathcal{C}_{\text {min }} \cup \mathcal{C}_{\text {max }}$ do not belong to $\mathcal{C}_{j} \backslash \mathcal{C}_{j+1}$, we know that $\hat{\lambda}_{i}=\lambda_{i}$ for all $i \in \mathcal{C}_{j} \backslash \mathcal{C}_{j+1}$, and thus

$$
\sum_{l=1}^{j} \sum_{i \in \mathcal{C}_{j} \backslash \mathcal{C}_{j+1}} x_{i, l}=\sum_{l=1}^{j} \sum_{i \in \mathcal{C}_{j} \backslash \mathcal{C}_{j+1}} \hat{x}_{i, l}
$$

from which we obtain

$$
\sum_{l<j} \sum_{i \in \mathcal{C}_{j} \backslash \mathcal{C}_{j+1}} x_{i, l}=\sum_{l<j} \sum_{i \in \mathcal{C}_{j} \backslash \mathcal{C}_{j+1}} \hat{x}_{i, l}+\left(\sum_{i \in \mathcal{C}_{j} \backslash \mathcal{C}_{j+1}} \hat{x}_{i, j}-\sum_{i \in \mathcal{C}_{j} \backslash \mathcal{C}_{j+1}} x_{i, j}\right),
$$

and therefore

$$
\sum_{l<j} \sum_{i \in \mathcal{C}_{j} \backslash \mathcal{C}_{j+1}} x_{i, l}<\sum_{l<j} \sum_{i \in \mathcal{C}_{j} \backslash \mathcal{C}_{j+1}} \hat{x}_{i, l}
$$

Substracting (21) from (20), we obtain

$$
\sum_{i \in \mathcal{C}_{j} \backslash \mathcal{C}_{j+1}} \hat{\mu}_{i}<\sum_{i \in \mathcal{C}_{j} \backslash \mathcal{C}_{j+1}} \mu_{i}
$$

Hence, for each server $l<j$,

$$
\left(N_{j}-N_{j+1}\right) \frac{c_{l}}{r_{l}-\hat{y}_{l}}+\frac{c_{l}}{\left(r_{l}-\hat{y}_{l}\right)^{2}} \sum_{i \in \mathcal{C}_{j} \backslash \mathcal{C}_{j+1}} \hat{x}_{i, l}<\left(N_{j}-N_{j+1}\right) \frac{c_{l}}{r_{l}-y_{l}}+\frac{c_{l}}{\left(r_{l}-y_{l}\right)^{2}} \sum_{i \in \mathcal{C}_{j} \backslash \mathcal{C}_{j+1}} x_{i, l} .
$$


But, for $l<j$ and $l \in \mathcal{S}^{+}$, it implies that

$$
\sum_{i \in \mathcal{C}_{j} \backslash \mathcal{C}_{j+1}} \hat{x}_{i, l}<\sum_{i \in \mathcal{C}_{j} \backslash \mathcal{C}_{j+1}} x_{i, l}
$$

and thus

$$
\sum_{l<j, l \in \mathcal{S}^{+}} \sum_{i \in \mathcal{C}_{j} \backslash \mathcal{C}_{j+1}} \hat{x}_{i, l}<\sum_{l<j, l \in \mathcal{S}^{+}} \sum_{i \in \mathcal{C}_{j} \backslash \mathcal{C}_{j+1}} x_{i, l}
$$

From (22), we have

$\sum_{l<j, l \in \mathcal{S}^{-}} \sum_{i \in \mathcal{C}_{j} \backslash \mathcal{C}_{j+1}} \hat{x}_{i, l}>\sum_{l<j, l \in \mathcal{S}^{-}} \sum_{i \in \mathcal{C}_{j} \backslash \mathcal{C}_{j+1}} x_{i, l}+\left(\sum_{l<j, l \in \mathcal{S}^{+}} \sum_{i \in \mathcal{C}_{j} \backslash \mathcal{C}_{j+1}} x_{i, l}-\sum_{l<j, l \in \mathcal{S}^{+}} \sum_{i \in \mathcal{C}_{j} \backslash \mathcal{C}_{j+1}} \hat{x}_{i, l}\right)$,

and using (23) it leads to

$$
\sum_{l<j, l \in \mathcal{S}^{-}} \sum_{i \in \mathcal{C}_{j} \backslash \mathcal{C}_{j+1}} \hat{x}_{i, l}>\sum_{l<j, l \in \mathcal{S}^{-}} \sum_{i \in \mathcal{C}_{j} \backslash \mathcal{C}_{j+1}} x_{i, l}
$$

According to (21), for each server $l<j$,

$$
N_{j+1} \frac{c_{l}}{r_{l}-\hat{y}_{l}}+\frac{c_{l}}{\left(r_{l}-\hat{y}_{l}\right)^{2}} \sum_{i \in \mathcal{C}_{j+1}} \hat{x}_{i, l}>N_{j+1} \frac{c_{l}}{r_{l}-y_{l}}+\frac{c_{l}}{\left(r_{l}-y_{l}\right)^{2}} \sum_{i \in \mathcal{C}_{j+1}} x_{i, l} .
$$

But, for $l<j, l \in \mathcal{S}^{-}$, it implies that

$$
\sum_{i \in \mathcal{C}_{j+1}} \hat{x}_{i, l}>\sum_{i \in \mathcal{C}_{j+1}} x_{i, l}
$$

and thus

$$
\sum_{l<j, l \in \mathcal{S}^{-}} \sum_{i \in \mathcal{C}_{j+1}} \hat{x}_{i, l}>\sum_{l<j, l \in \mathcal{S}^{-}} \sum_{i \in \mathcal{C}_{j+1}} x_{i, l}
$$

Now, summing (25) and (24) gives

$$
\sum_{l<j, l \in \mathcal{S}^{-}} \sum_{i \in \mathcal{C}_{j}} \hat{x}_{i, l}>\sum_{l<j, l \in \mathcal{S}^{-}} \sum_{i \in \mathcal{C}_{j}} x_{i, l} .
$$

However, for each server $l \in \mathcal{S}^{-}$, we have $\hat{y}_{l} \leq y_{l}$ and thus $\sum_{l<j, l \in \mathcal{S}^{-}} \hat{y}_{l} \leq$ $\sum_{l<j, l \in \mathcal{S}^{-}} y_{l}$. Since, for $l<j, y_{l}$ can also be written as $y_{l}=\sum_{i \in \mathcal{C}_{j}} x_{i, l}+$ $\sum_{i \notin \mathcal{C}_{j}} x_{i, l}$, it yields

$$
\sum_{l<j, l \in \mathcal{S}^{-}} \sum_{i \notin \mathcal{C}_{j}} \hat{x}_{i, l} \leq \sum_{l<j, l \in \mathcal{S}^{-}} \sum_{i \notin \mathcal{C}_{j}} x_{i, l}+\left(\sum_{l<j, l \in \mathcal{S}^{-}} \sum_{i \in \mathcal{C}_{j}} x_{i, l}-\sum_{l<j, l \in \mathcal{S}^{-}} \sum_{i \in \mathcal{C}_{j}} \hat{x}_{i, l}\right),
$$


and using (26),

$$
\sum_{l<j, l \in \mathcal{S}^{-}} \sum_{i \notin \mathcal{C}_{j}} \hat{x}_{i, l}<\sum_{l<j, l \in \mathcal{S}^{-}} \sum_{i \notin \mathcal{C}_{j}} x_{i, l} .
$$

Therefore, there exists a class $i \notin \mathcal{C}_{j}$ such that

$$
\sum_{l<j, l \in \mathcal{S}^{-}} \hat{x}_{i, l}<\sum_{l<j, l \in \mathcal{S}^{-}} x_{i, l}
$$

It implies that, for this class $i$, we can find a server $n \notin \mathcal{S}_{i}$ and $n \in \mathcal{S}^{-}$such that $\hat{x}_{i, n}<x_{i, n}$. Since $\mathcal{C}_{\text {max }} \subsetneq \mathcal{C}_{j}$, we know that $i \notin \mathcal{C}_{\text {max }}$. Moreover, since $\mathcal{S}_{k}=\mathcal{S}_{1} \subset \mathcal{S}^{+}$for all $k \in \mathcal{C}_{\text {min }}, i \notin \mathcal{C}_{\text {min }}$. We therefore have $\hat{\lambda}_{i}=\lambda_{i}$. Thus,

$$
\sum_{l \in \mathcal{S}^{-}} \hat{x}_{i, l}+\sum_{l \in \mathcal{S}^{+}} \hat{x}_{i, l}=\sum_{l \in \mathcal{S}^{-}} x_{i, l}+\sum_{l \in \mathcal{S}^{+}} x_{i, l},
$$

which implies

$$
\sum_{l \in \mathcal{S}^{+}} \hat{x}_{i, l}=\sum_{l \in \mathcal{S}^{+}} x_{i, l}+\left(\sum_{l \in \mathcal{S}^{-}} x_{i, l}-\sum_{l \in \mathcal{S}^{-}} \hat{x}_{i, l}\right),
$$

and with (28), it yields

$$
\sum_{l \in \mathcal{S}^{+}} \hat{x}_{i, l}>\sum_{l \in \mathcal{S}^{+}} x_{i, l}
$$

This implies that there exists a server $m<j, m \in \mathcal{S}^{+}$such that $\hat{x}_{i, m}>x_{i, m}$. But, according to Lemma 11, there cannot be two servers $m, n \in \mathcal{S}$ such that $\hat{y}_{m}>y_{m}, \hat{y}_{n} \leq y_{n}, \hat{x}_{i, m}>x_{i, m}$ and $\hat{x}_{i, n}<x_{i, n}$. This is a contradiction. Therefore, if $j \in \mathcal{S}^{-}$, then $j+1 \in \mathcal{S}^{-}$for all servers $j \in \mathcal{S}$.

Proposition 6 proves that the transformation induces a monotonic partition of servers: there exists a threshold $J<S$ such that for all servers $j>J$ the load is non-increasing under the transformation.

Using the above results regarding the impact of the transformation on the server loads, the following two theorems compare the costs $D(\boldsymbol{\lambda})$ and $D(\hat{\boldsymbol{\lambda}})$. The first theorem uses the following lemma.

Lemma 17 If $b_{i}, i=1,2, \ldots$, is such that
1. $b_{1}>0$,
2. $b_{i} \leq 0 \Rightarrow b_{i+1} \leq 0$, and
3. $\sum_{i} b_{i}=0$, 
and $a_{i}, i=1,2, \ldots$, is such that

1. $a_{i} \geq a_{i+1}$, and

2. $a_{I}-a_{I+1}>0$,

where $I=\max \left\{i: b_{i}>0\right\}$, then $\sum_{i} a_{i} b_{i}>0$.

Proof. We have

$$
\begin{aligned}
\sum_{i} a_{i} b_{i} & =\sum_{i \leq I} a_{i} b_{i}+\sum_{i>I} a_{i} b_{i} \\
& \geq a_{I} \sum_{i \leq I} b_{i}+\sum_{i>I} a_{i} b_{i} \\
& \geq a_{I} \sum_{i \leq I} b_{i}-a_{I+1} \sum_{i>I}\left|b_{i}\right| \\
& \geq\left(a_{I}-a_{I+1}\right) \sum_{i \leq I} b_{i} \\
& >0
\end{aligned}
$$

We are now in position to state our main results.

Theorem $4 D(\boldsymbol{\lambda})<D(\hat{\boldsymbol{\lambda}}) \Longleftrightarrow \mathcal{S}_{1} \subsetneq \mathcal{S}_{K}$.

Proof. We first show that if $\mathcal{S}_{1} \subsetneq \mathcal{S}_{K}$ then $D(\boldsymbol{\lambda})<D(\hat{\boldsymbol{\lambda}})$. As a function of the loads, the global cost is given by

$$
D(\boldsymbol{\lambda})=\sum_{j \in \mathcal{S}} \frac{c_{j} r_{j}}{r_{j}-y_{j}}-\sum_{j \in \mathcal{S}} c_{j} .
$$

Let $\Delta y_{j}=\hat{y}_{j}-y_{j}$. Note that $\Delta y_{j}>0 \Longleftrightarrow\left(r_{j}-\hat{y}_{j}\right)^{-1}>\left(r_{j}-y_{j}\right)^{-1}$, which leads to $\Delta y_{j} \neq 0 \Longleftrightarrow \Delta y_{j}\left(r_{j}-\hat{y}_{j}\right)^{-1}>\Delta y_{j}\left(r_{j}-y_{j}\right)^{-1}$. Thus,

$$
D(\hat{\boldsymbol{\lambda}})-D(\boldsymbol{\lambda})=\sum_{j \in \mathcal{S}} \frac{c_{j} r_{j}\left(\hat{y}_{j}-y_{j}\right)}{\left(r_{j}-\hat{y}_{j}\right)\left(r_{j}-y_{j}\right)} \geq \sum_{j \in \mathcal{S}} \frac{c_{j} r_{j}}{\left(r_{j}-y_{j}\right)^{2}} \Delta y_{j} .
$$

We now show that the RHS in the above inequality is positive. Since $\mathcal{S}_{1} \subsetneq$ $\mathcal{S}_{K}$, from Proposition 4 and Lemma 16, we can infer that $\mathcal{S}^{+} \neq \emptyset$ and $\mathcal{S}^{-} \neq \emptyset$. From Proposition 4, we can also infer that $\mathcal{S}_{1} \subset \mathcal{S}^{+}$. Hence, $\Delta y_{1}>0$. From Proposition 6 , if $j \in \mathcal{S}^{-}$then $j+1 \in \mathcal{S}^{-}$. Therefore, the sequence $\Delta y_{j}, j \in \mathcal{S}$ is such that

1. $\Delta y_{1}>0$, 
2. $\Delta y_{j} \leq 0 \Rightarrow \Delta y_{j+1} \leq 0$, and

3. $\sum_{j \in \mathcal{S}} \Delta y_{j}=0$.

Let $J=\max \left\{j: j \in \mathcal{S}^{+}\right\}$. Then, $J+1=\min \left\{j: j \in \mathcal{S}^{-}\right\}$. Note that $\mathcal{C}_{J} \neq \mathcal{C}_{J+1}$, otherwise from Lemma 13 , either both $J$ and $J+1$ belong to $\mathcal{S}^{+}$or both belong to $\mathcal{S}^{-}$. From Lemma 9 , we can conclude that

1. $\frac{c_{j} r_{j}}{\left(r_{j}-y_{j}\right)^{2}} \geq \frac{c_{j+1} r_{j+1}}{\left(r_{j+1}-y_{j+1}\right)^{2}}, \forall j$, and

2. $\frac{c_{J} r_{J}}{\left(r_{J}-y_{J}\right)^{2}}>\frac{c_{J+1} r_{J+1}}{\left(r_{J+1}-y_{J+1}\right)^{2}}$.

Since the sequences $c_{j} r_{j} /\left(\left(r_{j}-y_{j}\right)^{2}\right.$ and $\Delta y_{j}$ satisfy the conditions of Lemma 17 , we have

$$
\sum_{j} \frac{c_{j} r_{j}}{\left(r_{j}-y_{j}\right)^{2}} \Delta y_{j}>0
$$

and hence, from (30), we can conclude that $D(\boldsymbol{\lambda})<D(\hat{\boldsymbol{\lambda}})$.

To show the converse, if $D(\boldsymbol{\lambda})<D(\hat{\boldsymbol{\lambda}})$ then necessarily there exists an $m$ such that $y_{m} \neq \hat{y}_{m}$. From Proposition 4 , we obtain $\mathcal{S}_{1} \neq \mathcal{S}_{K}$. Since $\mathcal{S}_{1} \subset \mathcal{S}_{K}$, we can conclude that $\mathcal{S}_{1} \subsetneq \mathcal{S}_{K}$.

Remark 3 If $\mathcal{S}_{1} \subsetneq \mathcal{S}_{K}$, we have shown that in order to prove $D(\boldsymbol{\lambda})<D(\hat{\boldsymbol{\lambda}})$ it is sufficient to prove that $\sum_{j} \frac{c_{j} r_{j}}{\left(r_{j}-y_{j}\right)^{2}} \Delta y_{j}>0$. It is remarkable that it amounts to prove that $\sum_{j} \frac{\partial D}{\partial y_{j}} \Delta y_{j}>0$. However, the above proof is not based on this variational argument and is valid whatever the value of $0<\epsilon \leq n_{\max } \lambda_{K}$.

Theorem 4 shows that if all the classes do not use the same set of servers at the equilibrium $\mathbf{x}$, then the transformation will strictly increase the cost. The following theorem proves that the cost is constant under the transformation if all classes use the same set of servers.

Theorem $5 D(\boldsymbol{\lambda})=D(\hat{\boldsymbol{\lambda}}) \Longleftrightarrow \mathcal{S}_{1}=\mathcal{S}_{K}$.

Proof. From Lemma 16 and Proposition 4 , if $\mathcal{S}_{1}=\mathcal{S}_{K}$ then $y_{j}=\hat{y}_{j}, \forall j \in \mathcal{S}$ and therefore, $D(\boldsymbol{\lambda})=D(\hat{\boldsymbol{\lambda}})$. To prove the inverse, if $\mathcal{S}_{1} \neq \mathcal{S}_{K}$ then necessarily $\mathcal{S}_{1} \subsetneq \mathcal{S}_{K}$. From Theorem 4 , we can conclude that $D(\boldsymbol{\lambda}) \neq D(\hat{\boldsymbol{\lambda}})$.

\subsection{Maximum Step of a Basic Transformation}

Theorems 4 and 5 enable the comparison of the equilibria induced by two different rate vectors $\boldsymbol{\lambda}$ and $\hat{\boldsymbol{\lambda}}$, provided that $\hat{\boldsymbol{\lambda}}$ can be obtained from $\boldsymbol{\lambda}$ under a basic 
transformation which leaves unaffected the set of servers used by each class. The main limitation of these results comes from the latter assumption. However, as will be shown below, the continuity of the Nash mapping can be exploited to prove that, under certain conditions, the global cost is non-decreasing under the transformation even if some classes change the set of servers they use.

Definition 2 For each rate vector $\boldsymbol{\lambda} \in \Lambda$, the maximum step of the transformation $h_{\boldsymbol{\lambda}}$ is

$$
\Delta=\min \left(n_{\min } \Delta_{\min }, n_{\max } \Delta_{\max }\right)
$$

where $\Delta_{\min }=-\lambda_{1}+\min \left(\frac{\bar{\lambda}}{K}, \min _{i \in \mathcal{C} \backslash \mathcal{C}_{\min }} \lambda_{i}\right)$ and $\Delta_{\max }=\lambda_{K}-\max \left(\frac{\bar{\lambda}}{K}, \max _{i \in \mathcal{C} \backslash \mathcal{C}_{\max }} \lambda_{i}\right)$.

Intuitively, if the step $\epsilon$ of a basic transformation is lower than the maximum step $\Delta$, then the sets $\mathcal{C}_{\min }$ and $\mathcal{C}_{\max }$ will be unaffected by the transformation. On the contrary, if $\epsilon=\Delta$, then, after the transformation, we will have either (i) one more class in the set $\mathcal{C}_{\min }$ or $\mathcal{C}_{\max }$, or (ii) $\boldsymbol{\lambda}=\boldsymbol{\lambda}^{=}$.

For each rate vector $\boldsymbol{\lambda}$, let $\boldsymbol{\lambda}(\epsilon)=h_{\boldsymbol{\lambda}}(\epsilon)$ for $\epsilon \in[0, \Delta]$. All quantities of interest can be treated as functions of $\epsilon$. Therefore, in the following, if $z$ is a certain quantity related to the Nash equilibrium induced by the vector $\boldsymbol{\lambda}$ then we shall denote the corresponding quantity for vector $\boldsymbol{\lambda}(\epsilon)$ by $z(\epsilon)$.

We first prove the following properties of the transformation when $\epsilon \leq \Delta$.

Lemma 18 For each $\epsilon \leq \Delta$,

1. $\lambda_{i}(\epsilon) \leq \min \left(\frac{\bar{\lambda}}{K}, \min _{k \in \mathcal{C} \backslash \mathcal{C}_{m i n}} \lambda_{k}\right)$ for all $i \in \mathcal{C}_{\text {min }}$, and the inequality is strict if $\epsilon<n_{\min } \Delta_{\text {min }}$ whereas it holds as an equality if $\epsilon=\Delta=$ $n_{\min } \Delta_{\min }$

2. $\lambda_{i}(\epsilon) \geq \max \left(\frac{\bar{\lambda}}{K}, \max _{k \in \mathcal{C} \backslash \mathcal{C}_{\max }} \lambda_{k}\right)$ for all $i \in \mathcal{C}_{\max }$, and the inequality is strict if $\epsilon<n_{\max } \Delta_{\max }$, whereas it holds as an equality if $\epsilon=\Delta=$ $n_{\max } \Delta_{\max }$.

Proof. For $i \in \mathcal{C}_{\text {min }}$, we have $\lambda_{i}(\epsilon)=\lambda_{1}+\frac{\epsilon}{n_{m i n}}$. Since $\epsilon \leq n_{\text {min }} \Delta_{\text {min }}$, it yields $\lambda_{i}(\epsilon) \leq \lambda_{1}+\Delta_{m i n}$, i.e. $\lambda_{i}(\epsilon) \leq \min \left(\frac{\bar{\lambda}}{K}, \min _{k \in \mathcal{C} \backslash \mathcal{C}_{m i n}} \lambda_{k}\right)$, as claimed. Note that the inequality is strict if $\epsilon<n_{\min } \Delta_{\text {min }}$ and that it holds as an equality if $\epsilon=n_{\min } \Delta_{\min }$. The proof of property 2 is symmetric.

The following two lemmata detail how the sets $\mathcal{C}_{\min }$ and $\mathcal{C}_{\max }$ evolve under the transformation. 
Lemma 19 For each $\epsilon \leq \Delta$,

1. $\mathcal{C}_{\text {min }} \subseteq \mathcal{C}_{\min }(\epsilon)$ and $\mathcal{C}_{\max } \subseteq \mathcal{C}_{\max }(\epsilon)$,

2. If $\epsilon<n_{\min } \Delta_{\text {min }}$, then $\mathcal{C}_{\text {min }}=\mathcal{C}_{\text {min }}(\epsilon)$,

3. If $\epsilon<n_{\max } \Delta_{\max }$, then $\mathcal{C}_{\max }=\mathcal{C}_{\max }(\epsilon)$,

4. If $\epsilon=n_{\text {min }} \Delta_{\text {min }}$ and $\mathcal{C}_{\text {min }} \neq \mathcal{C}$, then $\mathcal{C}_{\text {min }} \subsetneq \mathcal{C}_{\text {min }}(\epsilon)$,

5. If $\epsilon=n_{\max } \Delta_{\max }$ and $\mathcal{C}_{\max } \neq \mathcal{C}$, then $\mathcal{C}_{\max } \subsetneq \mathcal{C}_{\max }(\epsilon)$.

Proof. We just prove the relations between $\mathcal{C}_{\min }$ and $\mathcal{C}_{\text {min }}(\epsilon)$, since the proofs of the relations between $\mathcal{C}_{\max }$ and $\mathcal{C}_{\max }(\epsilon)$ are symmetric. We first prove assertion 1. Let $i \in \mathcal{C}_{\text {min }}$. From Lemma 18.1, we have $\lambda_{i}(\epsilon) \leq \lambda_{k}$ for all $k \in \mathcal{C} \backslash \mathcal{C}_{\text {min }}$. For $k \notin \mathcal{C}_{\text {min }} \cup \mathcal{C}_{\max }$, Lemma 14.1 states that $\lambda_{k}(\epsilon)=\lambda_{k}$, which implies that $\lambda_{i}(\epsilon) \leq \lambda_{k}(\epsilon)$ for all $k \notin \mathcal{C}_{\text {min }} \cup \mathcal{C}_{\max }$. From Lemma 18.1, we also have $\lambda_{i}(\epsilon) \leq$ $\bar{\lambda} \frac{\bar{\lambda}}{K} \leq \lambda_{k}(\epsilon)$ for all $k \in \mathcal{C}_{\text {max }}$, where the last inequality comes from Lemma 18.2. We therefore conclude that $\lambda_{i}(\epsilon) \leq \lambda_{k}(\epsilon)$ for all $k \in \mathcal{C} \backslash \mathcal{C}_{\text {min }}$. However, from Lemma 14.2, we have $\lambda_{k}(\epsilon)=\lambda_{i}(\epsilon)=\lambda_{1}(\epsilon)$ for all $k \in \mathcal{C}_{\text {min }}$. We conclude that if $i \in \mathcal{C}_{\text {min }}$, then $\lambda_{i}(\epsilon) \leq \lambda_{k}(\epsilon)$ for all $k \in \mathcal{C}$, and thus $i \in \mathcal{C}_{\text {min }}(\epsilon)$. This shows that $\mathcal{C}_{\text {min }} \subset \mathcal{C}_{\text {min }}(\epsilon)$.

Let us now prove assertion 2. Assume $\epsilon<n_{\min } \Delta_{\text {min }}$. Since $\mathcal{C}_{\min } \subset \mathcal{C}_{\min }(\epsilon)$, we just need to prove that $\mathcal{C}_{\text {min }}(\epsilon) \subset \mathcal{C}_{\text {min }}$. It is sufficient to show that if $k \notin \mathcal{C}_{\text {min }}$, then $k \notin \mathcal{C}_{\text {min }}(\epsilon)$. Let $k \in \mathcal{C} \backslash \mathcal{C}_{\text {min }}$. If $k \notin \mathcal{C}_{\text {max }}$, then, according to Lemma 18.1, $\lambda_{1}(\epsilon)<\lambda_{k}=\lambda_{k}(\epsilon)$, whereas if $k \in \mathcal{C}_{\text {max }}, \lambda_{1}(\epsilon)<\frac{\bar{\lambda}}{K} \leq \lambda_{k}(\epsilon)$, also from Lemma 18. Since $\lambda_{1}(\epsilon)=\min _{i \in \mathcal{C}} \lambda_{i}(\epsilon)$, we conclude that $k \notin \mathcal{C}_{\min }(\epsilon)$, and thus that $\mathcal{C}_{\min }=\mathcal{C}_{\min }(\epsilon)$.

We now prove assertion 4. From Lemma 18.1, we have either $\lambda_{1}(\epsilon)=\frac{\bar{\lambda}}{K}$ or $\lambda_{1}(\epsilon)=\min _{k \in \mathcal{C} \backslash \mathcal{C}_{\min }} \lambda_{k}$.

- If $\lambda_{1}(\epsilon)=\frac{\bar{\lambda}}{K}$, then it clearly implies that $\lambda_{i}(\epsilon)=\frac{\bar{\lambda}}{K}$ for all $i \in \mathcal{C}$. However, since $\mathcal{C}_{\text {min }} \neq \mathcal{C}$, it implies that class $K$ belongs to $\mathcal{C}_{\text {min }}(\epsilon)$ but not to $\mathcal{C}_{\text {min }}$.

- If $\lambda_{1}(\epsilon)=\min _{k \in \mathcal{C} \backslash \mathcal{C}_{\text {min }}} \lambda_{k}$, we can find $j \in \mathcal{C} \backslash \mathcal{C}_{\text {min }}$ such that $\lambda_{j} \leq \frac{\bar{\lambda}}{K}$ and $\lambda_{j}=\min _{k \in \mathcal{C} \backslash \mathcal{C}_{\text {min }}} \lambda_{k}$. From $\lambda_{j} \leq \frac{\bar{\lambda}}{K}$ we deduce that $j \notin \mathcal{C}_{\text {max }}$. Therefore from Lemma 14.1 we obtain $\lambda_{j}(\epsilon)=\lambda_{j}=\lambda_{1}(\epsilon)$. We conclude that class $j$ belongs to $\mathcal{C}_{\min }(\epsilon)$ but not to $\mathcal{C}_{\min }$.

Since in both cases we can find a class $i \in \mathcal{C}_{\min }(\epsilon)$ such that $i \notin \mathcal{C}_{\text {min }}$, we conclude that $\mathcal{C}_{\text {min }} \subsetneq \mathcal{C}_{\text {min }}(\epsilon)$.

Lemma 20 The following statements hold. 
1. If $\mathcal{C}_{\text {min }} \cup \mathcal{C}_{\text {max }} \neq \mathcal{C}$, then $\mathcal{C}_{\text {min }} \cup \mathcal{C}_{\text {max }} \subsetneq \mathcal{C}_{\text {min }}(\Delta) \cup \mathcal{C}_{\text {max }}(\Delta)$.

2. If $\mathcal{C}_{\min } \cup \mathcal{C}_{\max }=\mathcal{C}$, then $\boldsymbol{\lambda}(\Delta)=\boldsymbol{\lambda}^{=}$.

Proof. We first prove assertion 1. Assume that $\mathcal{C}_{\min } \cup \mathcal{C}_{\max } \neq \mathcal{C}$. We have either $\Delta=n_{\min } \Delta_{\min }$, or $\Delta=n_{\max } \Delta_{\max }$. According to Lemmata 19.4 and 19.5, if $\Delta=n_{\min } \Delta_{\min }$, then $\mathcal{C}_{\min } \subsetneq \mathcal{C}_{\min }(\epsilon)$, while if $\Delta=n_{\max } \Delta_{\max }$ we have $\mathcal{C}_{\text {max }} \subsetneq \mathcal{C}_{\text {max }}(\epsilon)$. We therefore conclude that $\mathcal{C}_{\text {min }} \cup \mathcal{C}_{\text {max }} \subsetneq \mathcal{C}_{\text {min }}(\Delta) \cup \mathcal{C}_{\text {max }}(\Delta)$.

Now we prove assertion 2. We first note that if $\mathcal{C}_{\text {min }}=\mathcal{C}_{\text {max }}=\mathcal{C}$, then $\boldsymbol{\lambda}=\boldsymbol{\lambda}^{=}$, and thus $\boldsymbol{\lambda}(\Delta)=\boldsymbol{\lambda}^{=}$. So we consider the case $\mathcal{C}_{\min } \neq \mathcal{C}_{\max }$. Since $\mathcal{C} \backslash \mathcal{C}_{\text {min }}=\mathcal{C}_{\text {max }}, \Delta_{\text {min }}=-\lambda_{1}+\min \left(\bar{\lambda} / K, \min _{i \in \mathcal{C}_{\text {max }}} \lambda_{i}\right)=\bar{\lambda} / K-\lambda_{1}$. Similarly, $\Delta_{\max }=\lambda_{K}-\bar{\lambda} / K$.

Also, $n_{\min } \Delta_{\min }=n_{\min } \bar{\lambda} / K-n_{\min } \lambda_{1}=\left(K-n_{\max }\right) \bar{\lambda} / K-\left(\bar{\lambda}-n_{\max } \lambda_{K}\right)=$ $n_{\max } \Delta_{\max }$. Hence, $n_{\min } \Delta_{\min }=n_{\max } \Delta_{\max }=\Delta$. Now, $\forall i \in \mathcal{C}_{\min }, \lambda_{i}(\Delta)=$ $-\lambda_{1}+\Delta / n_{\min }=\bar{\lambda} / K$. Similarly, $\forall i \in \mathcal{C}_{\max }, \lambda_{i}(\Delta)=\bar{\lambda} / K$. Hence, $\boldsymbol{\lambda}(\Delta)=\boldsymbol{\lambda}^{=}$.

The following proposition states that if we consider two rate vectors obtained from $\boldsymbol{\lambda}$ under basic transformations of steps lower than the maximum step, then one can be obtained from the other by a basic transformation.

Proposition 7 Let $\epsilon_{1}, \epsilon_{2} \in[0, \Delta], \epsilon_{1}<\epsilon_{2}$. Then $\boldsymbol{\lambda}\left(\epsilon_{2}\right)$ can be obtained from $\boldsymbol{\lambda}\left(\epsilon_{1}\right)$ under a basic transformation.

Proof. Since $\epsilon_{1}<\epsilon_{2}$ implies $\epsilon_{1}<\Delta$, from Lemmata 19.2 and 19.3 we have $\mathcal{C}_{\text {min }}\left(\epsilon_{1}\right)=\mathcal{C}_{\text {min }}$ and $\mathcal{C}_{\text {max }}\left(\epsilon_{1}\right)=\mathcal{C}_{\text {max }}$. Accordingly, $\boldsymbol{\lambda}\left(\epsilon_{2}\right)$ can be written as

$$
\begin{aligned}
\boldsymbol{\lambda}\left(\epsilon_{2}\right)= & \boldsymbol{\lambda}+\epsilon_{1}\left(\frac{\sum_{i \in \mathcal{C}_{\min }} \mathbf{e}_{i} \sum_{i \in \mathcal{C}_{\max }} \mathbf{e}_{i}}{n_{\min }}-\frac{\sum_{\max }}{\mathbf{e}_{i}} \sum_{i \in \mathcal{C}_{\max }\left(\epsilon_{1}\right)} \mathbf{e}_{i}\right. \\
& +\left(\epsilon_{2}-\epsilon_{1}\right) \\
n_{\max }\left(\epsilon_{1}\right) & \left(\frac{i \in \mathcal{C}_{\min }\left(\epsilon_{1}\right)}{n_{\min }\left(\epsilon_{1}\right)}-\right.
\end{aligned}
$$

i.e., $\boldsymbol{\lambda}\left(\epsilon_{2}\right)=h_{\boldsymbol{\lambda}\left(\epsilon_{1}\right)}\left(\epsilon_{2}-\epsilon_{1}\right)$.

We now show that even if some classes change the set of servers they use, the global cost is non-decreasing under the transformation $\boldsymbol{\lambda}(\epsilon)=h_{\boldsymbol{\lambda}}(\epsilon)$ provided that $\epsilon \leq \Delta$. The proof is based on the following theorem which is proved in [14] (Theorem 5, page 321), and closely parallels the discussion in section III.B of the above reference. 
Theorem 6 (Theorem 5 in [14]) Let $f: X \rightarrow \mathbb{R}$, where $X \subset \mathbb{R}$ is a closed interval. Consider a family $\mathcal{A}=\left\{A_{1}, \ldots, A_{n}\right\}$ of closed subsets of $X$, such that (i) $\cup_{i=1}^{n} A_{i}=X$, and (ii) for every $A_{i} \in \mathcal{A}$, we have : $x, y \in A_{i}$ and $x<y \Rightarrow f(x)<f(y)$. Then $f$ is non-decreasing in $X$.

The following theorem extends Theorems 4 and 5 to the case when the transformation changes the set of servers used by some classes.

Theorem 7 For $\epsilon \leq \Delta, D(\boldsymbol{\lambda}(\epsilon)) \geq D(\boldsymbol{\lambda})$.

Proof. Consider a rate vector $\boldsymbol{\lambda}$ and the transformation $\boldsymbol{\lambda}(\epsilon)=h_{\boldsymbol{\lambda}}(\epsilon)$ of this rate vector for $\epsilon \in[0, \Delta]$. We want to prove that $D(\boldsymbol{\lambda}(\epsilon)) \geq D(\boldsymbol{\lambda})$. Since all quantities of interest, and in particular the global cost, can be treated as functions of $\epsilon$, it suffices to show that $D$ is a nondecreasing function of $\epsilon$ on $[0, \Delta]$.

Let $A_{i, j}=\left\{\epsilon \in[0, \Delta]: G_{i, j}(\epsilon) \leq \lambda_{i}(\epsilon) \leq G_{i, j+1}(\epsilon)\right\}$, denote the set of $\epsilon \epsilon$ $[0, \Delta]$ for which class $i$ sends flow to servers $\{1, \ldots, j\}$ under equilibrium $\mathcal{N}(\epsilon)$. From (13), one can see that $G_{i, j}$ is a continuous function of the $r_{i, j}$, which in turn are continuous function of the equilibrium strategies of the other classes. The continuity of the Nash mapping then implies that $G_{i, j}$ is a continuous function of $\epsilon \in[0, \Delta]$. Continuity of the functions $G_{i, j}(\epsilon)$ and $\lambda_{i}(\epsilon)$ implies that $A_{i, j}$ is a closed set.

For each $\mathbf{S} \in \mathcal{S}^{K}$, define

$$
A_{\mathbf{S}}=\cap_{i \in \mathcal{C}} A_{i, S_{i}}
$$

which is also a closed set. If $\epsilon_{1}, \epsilon_{2} \in A_{\mathbf{S}}$, then each class sends its flow to the same set of servers under equilibria $\mathcal{N}\left(\epsilon_{1}\right)$ and $\mathcal{N}\left(\epsilon_{2}\right)$.

Consider a vector $\mathbf{S} \in \mathcal{S}^{K}$ and assume that we can find $\epsilon_{1}, \epsilon_{2} \in A_{\mathbf{S}}$ such that $\epsilon_{1}<\epsilon_{2}$, i.e. $A_{\mathbf{S}}$ is neither empty nor reduced to an isolated point. According to Proposition 7 , the vector $\boldsymbol{\lambda}\left(\epsilon_{2}\right)$ can be obtained from $\boldsymbol{\lambda}\left(\epsilon_{1}\right)$ under a basic transformation. Since $\epsilon_{1}, \epsilon_{2} \in A_{\mathbf{S}}$, this transformation satisfies Assumption 1, and according to Theorems 4 and 5 we have either $D\left(\epsilon_{2}\right)>D\left(\epsilon_{1}\right)$ or $D\left(\epsilon_{2}\right)=$ $D\left(\epsilon_{1}\right)$. We therefore conclude that if we can find $\epsilon_{1}, \epsilon_{2} \in A_{\mathbf{S}}$ such that $\epsilon_{1}<\epsilon_{2}$, then $D\left(\epsilon_{2}\right) \geq D\left(\epsilon_{1}\right)$.

Since $[0, \Delta]=\cup_{\mathbf{S} \in \mathcal{S}^{K}} A_{\mathbf{S}}$, all conditions of Theorem 6 are fullfilled, and we can conclude that $D$ is a nondecreasing function of $\epsilon$ on $[0, \Delta]$.

\subsection{Maximum of the Global Cost}

The purpose of this section is to prove that the global cost achieves its maximum in the symmetric case, i.e., when $\boldsymbol{\lambda}=\boldsymbol{\lambda}^{=}=\left(\frac{\bar{\lambda}}{K}, \ldots, \frac{\bar{\lambda}}{K}\right)$. To this end, starting 
from a fixed rate vector $\boldsymbol{\lambda}$, we build a sequence $\left(\boldsymbol{\lambda}^{k}\right)_{k \in \mathbb{N}}$ of rate vectors such that:

- $\boldsymbol{\lambda}^{0}=\boldsymbol{\lambda}$, and

- $\boldsymbol{\lambda}^{k+1}$ is obtained from $\boldsymbol{\lambda}^{k}$ under a basic transformation of maximum step, i.e., $\boldsymbol{\lambda}^{k+1}=h_{\boldsymbol{\lambda}^{k}}\left(\Delta^{k}\right)$.

The following proposition shows that the sequence $\left(\boldsymbol{\lambda}^{k}\right)_{k \in \mathbb{N}}$ converges to $\boldsymbol{\lambda}^{=}$in a finite number of steps.

Proposition 8 The sequence $\left(\boldsymbol{\lambda}^{k}\right)_{k \in \mathbb{N}}$ converges to $\boldsymbol{\lambda}^{=}$in at most $K$ steps.

Proof. Let $w^{k}$ be the number of classes in $\mathcal{C}_{\min }^{k} \cup \mathcal{C}_{\max }^{k}$. Note that $w^{0} \geq 2$. According to Lemma 20.2, if $w^{k}=K$, then $\boldsymbol{\lambda}^{k+1}=\boldsymbol{\lambda}^{=}$. Otherwise, according to Lemma 20.1, we have $\mathcal{C}_{\text {min }}^{k} \cup \mathcal{C}_{\text {max }}^{k} \subsetneq \mathcal{C}_{\text {min }}^{k+1} \cup \mathcal{C}_{\text {max }}^{k+1}$, and thus $w^{k}<w^{k+1} \leq K$. This structure implies that in at most $K$ steps we have $w^{k}=K$, and thus $\lambda^{k+1}=\lambda^{=}$.

We now prove Theorem 1.

Proof of Theorem 1. For each $\boldsymbol{\lambda} \in \Lambda$, the sequence $\left(\boldsymbol{\lambda}^{k}\right)_{k \in \mathbb{N}}$ converges to $\boldsymbol{\lambda}^{=}$ in a finite number of steps. According to Theorem 7, we have $D\left(\boldsymbol{\lambda}^{k+1}\right) \geq D\left(\boldsymbol{\lambda}^{k}\right)$. This implies that $D\left(\boldsymbol{\lambda}^{=}\right) \geq D(\boldsymbol{\lambda})$.

\section{Price of Anarchy}

According to Theorem 1, we have

$$
P o A(K)=\sup _{\boldsymbol{\lambda}, \mathbf{r}, \mathbf{c}} \frac{D_{K}(\boldsymbol{\lambda}, \mathbf{r}, \mathbf{c})}{D_{1}(\bar{\lambda}, \mathbf{r}, \mathbf{c})}=\sup _{\mathbf{r}, \mathbf{c}} \frac{D_{K}\left(\boldsymbol{\lambda}^{=}, \mathbf{r}, \mathbf{c}\right)}{D_{1}(\bar{\lambda}, \mathbf{r}, \mathbf{c})}
$$

Therefore, in order to analyze the PoA, we can focus on the symmetric case. We analyze the symmetric game in Section 6.1 and derive an explicit expression for the equilibrium flows. These results are then used in Section 6.2 to prove that the PoA is upper-bounded by the square root of the number of dispatchers. In Section 6.3 we prove the lower bound on the PoA by exhibiting an example for which the ratio $\frac{D_{K}(\boldsymbol{\lambda}, \mathbf{r}, \mathbf{c})}{D_{1}(\lambda, \mathbf{r}, \mathbf{c})}$ is $K /(2 \sqrt{K}-1)$. Finally, in Section 6.4 , we summarize our result on the PoA and discuss its consequences. 


\subsection{Analysis of the Symmetric Game}

It is well known that in this case the non-cooperative routing game is a potential game, i.e., the equilibrium flows are the global minima of a standard convex optimization problem (see e.g. Theorem 4.1 in [7]). This is formally stated in the following proposition.

Proposition 9 If the vector $\mathbf{y}$ is global optimum of the following convex optimization problem, then

$$
\begin{array}{ll}
\underset{\mathbf{y}}{\operatorname{minimize}} & \sum_{j \in \mathcal{S}} \frac{c_{j}}{K}\left[\frac{y_{j}}{r_{j}-y_{j}}+(K-1) \log \left(\frac{r_{j}}{r_{j}-y_{j}}\right)\right] \\
\text { s.t. } & \sum_{j \in \mathcal{S}} y_{j}=\bar{\lambda}, \\
& 0 \leq y_{j}<r_{j}, \forall j \in \mathcal{S} .
\end{array}
$$

then the multi-strategy $\mathbf{x}=(\mathbf{y} / K, \ldots, \mathbf{y} / K)$ is a NEP of the symmetric game.

Proof. The statement follows from Theorem 4.1 in [7]) with $c_{a}\left(x_{a}\right)=\frac{c_{a}}{r_{a}-x_{a}}$.

Note that when $K=1$, the above problem reduces to the global optimization problem solved by the centralized scheme, whereas when $K \rightarrow \infty$, the above problem reduces to the problem stated in Proposition 4 of [1]. In the latter case, the equivalent problem states that the common function optimized jointly by an infinite number of players and is characteristic of the Wardrop equilibrium.

In order to describe the solution of the above equivalent problem, let us define $u_{j}=c_{j} / r_{j}, j \in \mathcal{S}$, and $u_{S+1}=\infty$. Note that, by definition, the sequence $u_{j}$ is increasing in $j$. Let us also define the function

$W_{j}(K, z)=\mathbb{1}_{\left\{z \in\left[u_{j}, u_{j+1}\right)\right\}} \cdot\left(\sum_{s=1}^{j} \frac{2 r_{s}}{\sqrt{(K-1)^{2}+4 K u_{s}^{-1} z}-(K-1)}-\sum_{s=1}^{j} r_{s}+\bar{\lambda}\right)$,

and let $W(K, z)=\sum_{j \in \mathcal{S}} W_{j}(K, z)$. The following lemma states some properties of the function $W(K, z)$.

Lemma 21 The function $W(K, z)$ is such that:

1. for a fixed $K$, the function $W:\left[u_{1}, \infty\right) \rightarrow \mathbb{R}$ is continuous and decreasing in $z$,

2. for a fixed $z, W(K, z)$ is decreasing in $K$,

3. for a fixed $K, W(K, z)=0$ has a unique solution in the interval $\left(u_{1}, \infty\right)$. 
Proof. Let us first prove property 1 . By definition $W(k, x)=W_{j}(K, x)$ in the interval $\left[u_{j}, u_{j+1}\right)$. Since $W_{j}$ is continuous and decreasing in $\left(u_{j}, u_{j+1}\right)$ so is $W$. To conclude the proof, we need to verify that $W$ is continuous at $u_{j}, j=2,3, \ldots, S$. We have

$$
\begin{aligned}
\lim _{x \rightarrow u_{j}^{+}} W(K, x)-\lim _{x \rightarrow u_{j}^{-}} W(K, x)= & \lim _{x \rightarrow u_{j}^{+}} W_{j}(K, x)-\lim _{x \rightarrow u_{j}^{-}} W_{j-1}\left(K, u_{j}\right) \\
= & \left(\sum_{i=1}^{j} \frac{2 r_{i}}{\sqrt{(K-1)^{2}+4 K u_{i}^{-1} u_{j}}-(K-1)}-\sum_{i=1}^{j} r_{i}+\bar{\lambda}\right) \\
& -\left(\sum_{i=1}^{j-1} \frac{2 r_{i}}{\sqrt{(K-1)^{2}+4 K u_{i}^{-1} u_{j}}-(K-1)}-\sum_{i=1}^{j-1} r_{i}+\bar{\lambda}\right) \\
= & 0,
\end{aligned}
$$

which shows that the function $W(K, x)$ is also continuous at the points $u_{j}, j=$ $2,3, \ldots, S$.

To prove property 2 , it is sufficient to show that for $K_{1}<K_{2}$,

$$
\frac{1}{\sqrt{\left(K_{1}-1\right)^{2}+4 K_{1} u_{i}^{-1} u_{j}}-\left(K_{1}-1\right)}>\frac{1}{\sqrt{\left(K_{2}-1\right)^{2}+4 K_{2} u_{i}^{-1} u_{j}}-\left(K_{2}-1\right)} \text {. }
$$

It is thus sufficient to show that

$$
\sqrt{\left(K_{2}-1\right)^{2}+4 K_{2} u_{i}^{-1} u_{j}}-\left(K_{2}-1\right)>\sqrt{\left(K_{1}-1\right)^{2}+4 K_{1} u_{i}^{-1} u_{j}}-\left(K_{1}-1\right) .
$$

We show below that, under a certain condition, the function $\sqrt{(K-1)^{2}+4 K u_{i}^{-1} u_{j}}-$ $(K-1)$ is increasing in $K$ on assuming $K$ to be real, and that this condition is satisfied. For this we show its derivative is positive, which is equivalent to showing

$$
\begin{array}{rlrl} 
& & \frac{1}{2} \frac{2(K-1)+4 u_{i}^{-1} u_{j}}{\sqrt{(K-1)^{2}+4 K u_{i}^{-1} u_{j}}}-1 & >0 \\
\Leftrightarrow & (K-1)+2 u_{i}^{-1} u_{j} & >\sqrt{(K-1)^{2}+4 K u_{i}^{-1} u_{j}} \\
\Leftrightarrow & (K-1)^{2}+4\left(u_{i}^{-1} u_{j}\right)^{2}+4(K-1) u_{i}^{-1} u_{j} & >(K-1)^{2}+4 K u_{i}^{-1} u_{j} \\
\Leftrightarrow & 4\left(u_{i}^{-1} u_{j}\right)^{2}-4 u_{i}^{-1} u_{j} & >0 .
\end{array}
$$

Since $u_{i}^{-1} u_{j}>1$ the above inequality holds.

Finally, let us now prove property 3 . First, we note that $W\left(K, u_{1}\right)=\bar{\lambda}$ and $W(K, \infty)=-\bar{r}+\bar{\lambda}$ which is negative (by assumption). Also according to property $1, W(K, x)$ is continuous and decreasing in the interval $\left[u_{1}, \infty\right)$. Hence, there is a unique value of $x$ for which $W(K, x)=0$.

In the following, we let $\gamma(K)$ be the unique solution of $W(K, x)=0$ in $\left[u_{1}, \infty\right)$. 
The following proposition gives the solution of the symmetric game.

Proposition 10 The subset of servers that are used at the NEP is $\mathcal{S}^{*}(K)=$ $\left\{1,2, \ldots, j^{*}(K)\right\}$, where $j^{*}(K)$ is the greatest value of $j$ such that $W\left(K, u_{j+1}\right) \leq$ $0<W\left(K, u_{j}\right)$. The equilibrium flows are $x_{i, j}=\frac{y_{j}}{K}, i \in \mathcal{C}, j \in \mathcal{S}^{*}(K)$, where the offered traffic of server $j$ is given by

$$
y_{j}=r_{j} \frac{\sqrt{(K-1)^{2}+4 K \gamma(K) r_{j} / c_{j}}-(K+1)}{\sqrt{(K-1)^{2}+4 K \gamma(K) r_{j} / c_{j}}-(K-1)},
$$

with $\gamma(K)$ the unique root of $W(K, z)=0$ in $\left[u_{1}, \infty\right)$.

Proof. Let $\mathbf{y}$ be an optimal solution of the equivalent problem stated in Proposition 9. According to the KKT conditions, there exist $\gamma$ and $\nu_{j} \geq 0, j \in \mathcal{S}$ such that for each $j \in \mathcal{S}$,

$$
\begin{aligned}
y_{j} \nu_{j} & =0 \\
\frac{u_{j}}{K} \phi_{j}\left(\phi_{j}+K-1\right) & =\gamma+\nu_{j}
\end{aligned}
$$

where $\phi_{j}=r_{j} /\left(r_{j}-y_{j}\right)$. Since $\nu_{j} \geq 0$, we have from (35) that

$$
K u_{j}^{-1} \gamma \leq \phi_{j}\left(\phi_{j}+K-1\right), \quad \forall j \in \mathcal{S}
$$

with equality if and only if $y_{j}>0$. Moreover, eliminating $\nu_{j}$ from (34), we obtain the following complementary slackness condition

$$
y_{j}\left[\phi_{j}\left(\phi_{j}+K-1\right)-K u_{j}^{-1} \gamma\right]=0, \quad \forall j \in \mathcal{S}
$$

Let us now consider a server $j$. Let us first assume that $u_{j}<\gamma$. In this case, a necessary condition for $(36)$ to hold is $\phi_{j}\left(\phi_{j}+K-1\right)>K$, which implies $\phi_{j}>1$ and hence $y_{j}>0$. We therefore obtain from (37) that

$$
\phi_{j}^{2}+(K-1) \phi_{j}-K u_{j}^{-1} \gamma=0
$$

The above equation has a single positive root given by

$$
\phi_{j}=\frac{1}{2}\left[\sqrt{(K-1)^{2}+4 K u_{j}^{-1} \gamma}-(K-1)\right] .
$$

We thus conclude that if $u_{j}<\gamma$, then the load $y_{j}=r_{j}\left(\phi_{j}-1\right) / \phi_{j}$ of the server $j$ is given by

$$
y_{j}=r_{j} \frac{\sqrt{(K-1)^{2}+4 K u_{j}^{-1} \gamma}-(K+1)}{\sqrt{(K-1)^{2}+4 K u_{j}^{-1} \gamma}-(K-1)} .
$$

Let us now assume on the contrary that $u_{j}>\gamma$. If $y_{j}>0$, then $\phi_{j}>1$, which implies that $\phi_{j}\left(\phi_{j}+K-1\right)>K$. However, according to the complementary 
slackness condition (37), the left hand side is just $K u_{j}^{-1} \gamma$, and we therefore obtain that $\gamma>u_{j}$, i.e., a contradiction. As a consequence, if $u_{j}>\gamma$, then $y_{j}=0$. Finally, we conclude from the above analysis that

$$
y_{j}= \begin{cases}r_{j} \frac{\sqrt{(K-1)^{2}+4 K u_{j}^{-1} \gamma}-(K+1)}{\sqrt{(K-1)^{2}+4 K u_{j}^{-1} \gamma}-(K-1)} & \text { if } u_{j}<\gamma \\ 0 & \text { otherwise. }\end{cases}
$$

Let $j^{*}(K)$ be such that $u_{j^{*}(K)}<\gamma \leq u_{j^{*}(K)+1}$. Then the subset of servers used at the Nash equilibrium is $\mathcal{S}^{*}(K)=\left\{1, \ldots, j^{*}(K)\right\}$. Using (38), we deduce from $\sum_{j \in \mathcal{S}^{*}(K)} y_{j}=\bar{\lambda}$ that

$$
\sum_{j \in \mathcal{S}^{*}(K)} r_{j}-\bar{\lambda}=\sum_{k \in \mathcal{S}^{*}(K)} \frac{2 r_{k}}{\sqrt{(K-1)^{2}+4 K u_{k}^{-1} \gamma}-(K-1)},
$$

i.e., $W(K, \gamma)=0$, which implies that $\gamma=\gamma(K)$ according to Lemma 21.3. Moreover, since for a fixed $K$ the function $W:\left[u_{1}, \infty\right) \rightarrow \mathbb{R}$ is decreasing in $z$, we deduce from $u_{j^{*}(K)}<\gamma(K) \leq u_{j^{*}(K)+1}$ that

$$
W\left(K, u_{j^{*}(K)+1}\right) \leq 0<W\left(K, u_{j^{*}(K)}\right) .
$$

We now prove that the distributed scheme with $K$ dispatchers uses only a subset of the servers used by the centralized scheme. The proof is based on the following proposition.

Proposition 11 The function $\gamma(K)$ is decreasing in $K$.

Proof. For $K_{1}<K_{2}$, we have $0=W\left(K_{1}, \gamma\left(K_{1}\right)\right)>W\left(K_{2}, \gamma\left(K_{1}\right)\right)$, where the inequality follows from Lemma 21.2. Using $W\left(K_{2}, u_{1}\right)=\bar{\lambda}>0$, and Lemma 21.3 , we can conclude that $u_{1}<\gamma\left(K_{2}\right)<\gamma\left(K_{1}\right)$.

The fact that $\gamma(K)$ is decreasing in $K$ implies that $j^{*}(K)$ is non-increasing in $K$. We therefore have the following important corollary.

Corollary 5 For $K \geq 1, \mathcal{S}^{*}(K+1) \subset \mathcal{S}^{*}(K)$.

As an immediate consequence, we can conclude that $\mathcal{S}^{*}(K) \subset \mathcal{S}^{*}(1)$, i.e., the distributed scheme with $K$ dispatchers uses only a subset of the servers used by the centralized scheme. 


\subsection{Upper Bound on the PoA}

In order to distinguish between the offered traffic in server $j$ for different values of $K$, we denote by $y_{j}(K)$ the offered traffic in equilibrium in the $K$ player symmetric game, where $y_{j}(K)$ is given by $(33)$.

The following lemma gives a bound on the mean number of jobs in a server in the decentralized case in terms of the mean number of jobs in the same server in the centralized case.

\section{Lemma 22}

$$
\frac{y_{j}(K)}{r_{j}-y_{j}(K)} \leq \sqrt{K} \frac{y_{j}(1)}{r_{j}-y_{j}(1)}, \forall j \in \mathcal{S}^{*}(1)
$$

Proof. From Corollary 5, we have $\mathcal{S}^{*}(K) \subset \mathcal{S}^{*}(1)$. For $j \in \mathcal{S}^{*}(1) \backslash \mathcal{S}^{*}(K)$, $\rho_{j}(K)=0$. Hence (39) holds. It now remains to be shown that (39) holds for every $j \in \mathcal{S}^{*}(K)$.

From (33),

$$
y_{j}(K)=r_{j} \frac{\sqrt{(K-1)^{2}+4 K \gamma(K) r_{j} / c_{j}}-(K+1)}{\sqrt{(K-1)^{2}+4 K \gamma(K) r_{j} / c_{j}}-(K-1)},
$$

from which it follows that

$$
1-\frac{y_{j}(K)}{r_{j}}=\frac{2}{\sqrt{(K-1)^{2}+4 K \gamma(K) r_{j} / c_{j}}-(K-1)},
$$

and that

$$
\frac{y_{j}(K)}{r_{j}-y_{j}(K)}=\frac{\sqrt{(K-1)^{2}+4 K \gamma(K) r_{j} / c_{j}}-(K+1)}{2} .
$$

We shall now use the fact that $y_{j}(K) /\left(r_{j}-y_{j}(K)\right)$ is increasing in $\gamma(K)$. Since 


$$
\begin{aligned}
\gamma(K) \leq \gamma(1), & \text { from }(42), \\
\frac{y_{j}(K)}{r_{j}-y_{j}(K)} & \leq \frac{\sqrt{(K-1)^{2}+4 K \gamma(1) r_{j} / c_{j}}-(K+1)}{2} \\
& =\frac{\sqrt{(K-1)^{2}+4 K \gamma(1) r_{j} / c_{j}}-(K+1)}{2} \frac{\sqrt{(K-1)^{2}+4 K \gamma(1) r_{j} / c_{j}}+(K+1)}{\sqrt{(K-1)^{2}+4 K \gamma(1) r_{j} / c_{j}}+(K+1)} \\
& =\frac{4 K \gamma(1) r_{j} / c_{j}-4 K}{2} \frac{1}{\sqrt{(K-1)^{2}+4 K \gamma(1) r_{j} / c_{j}}+(K+1)} \\
& =2 K \frac{\gamma(1) r_{j} / c_{j}-1}{\sqrt{(K-1)^{2}+4 K \gamma(1) r_{j} / c_{j}}+(K+1)} \\
& \leq 2 K \frac{\gamma(1) r_{j} / c_{j}-1}{\sqrt{4 K \gamma(1) r_{j} / c_{j}}+(K+1)} \quad(\text { since } K-1 \geq 0) \\
& \leq 2 K \frac{\gamma(1) r_{j} / c_{j}-1}{\sqrt{4 K \gamma(1) r_{j} / c_{j}}+2 \sqrt{K}} \quad(\text { since } K+1 \geq 2 \sqrt{K}) \\
& =\sqrt{K} \frac{\gamma(1) r_{j} / c_{j}-1}{\sqrt{\gamma(1) r_{j} / c_{j}}+1} \\
& =\sqrt{K}\left(\sqrt{\gamma(1) r_{j} / c_{j}}-1\right) .
\end{aligned}
$$

From (42),

$$
\frac{y_{j}(1)}{r_{j}-y_{j}(1)}=\frac{\sqrt{4 \gamma(1) r_{j} / c_{j}}-2}{2}=\sqrt{\gamma(1) r_{j} / c_{j}}-1,
$$

which upon substitution in (43) gives

$$
\frac{y_{j}(K)}{r_{j}-y_{j}(K)} \leq \sqrt{K} \frac{y_{j}(1)}{r_{j}-y_{j}(1)}, \forall j \in \mathcal{S}^{*}(K) .
$$

The above lemma leads to the following upper bound on $P o A(K)$.

\section{Proposition 12}

$$
\operatorname{Po} A(K) \leq \sqrt{K}
$$

Proof. Since $\mathcal{S}^{*}(K) \subset \mathcal{S}^{*}(1)$,

$$
\begin{aligned}
D_{K}\left(\boldsymbol{\lambda}^{=}, \mathbf{r}, \mathbf{c}\right) & =\sum_{j \in \mathcal{S}^{*}(K)} c_{j} \frac{y_{j}(K)}{r_{j}-y_{j}(K)} \\
& \leq \sum_{j \in \mathcal{S}^{*}(1)} c_{j} \frac{y_{j}(K)}{r_{j}-y_{j}(K)} .
\end{aligned}
$$


which, on substituting from Lemma 22, gives

$$
\frac{D_{K}\left(\boldsymbol{\lambda}^{=}, \mathbf{r}, \mathbf{c}\right)}{D_{1}(\bar{\lambda}, \mathbf{r}, \mathbf{c})} \leq \sqrt{K}
$$

Since this bound in independent of $\mathbf{r}$ and $\mathbf{c}$, we can conclude that $\operatorname{Po} A(K) \leq$ $\sqrt{K}$.

\subsection{Lower Bound on the PoA}

We now give an example which shows that the PoA is bounded below by $K /(2 \sqrt{K}-1)$.

\section{Proposition 13}

$$
\operatorname{PoA}(K) \geq \frac{K}{2 \sqrt{K}-1}
$$

Proof. To prove this statement, we give a particular choice of the $\mathbf{r}$ and $\mathbf{c}$ for which $\frac{D_{K}(\boldsymbol{\lambda}, \mathbf{r}, \mathbf{c})}{D_{1}(\lambda, \mathbf{r}, \mathbf{c})}=\frac{K}{2 \sqrt{K}-1}$, independently of the number of servers $S \geq 2$. It follows closely the example in Theorem 5 in [1]. We take $c_{j}=r_{j}=1$, for $j>1$. Using Proposition 3, one can verify that if

$$
\frac{\left(r_{1}-\bar{\lambda}\right)^{2}}{r_{1}}<c_{1}<\frac{\left(r_{1}-\bar{\lambda}\right)^{2}}{r_{1}-\bar{\lambda}+\frac{1}{K} \bar{\lambda}}
$$

then the centralized scheme will use all servers whereas, at the NEP, the distributed scheme with $K$ dispatchers will only use the first server. In order to ensure that (46) is always satisfied we set $c_{1}=\left(r_{1}-\bar{\lambda}\right)^{2} \alpha\left(r_{1}\right)$ for $\alpha\left(r_{1}\right)$ such that $r_{1}^{-1}<\alpha\left(r_{1}\right)<\left(r_{1}-\bar{\lambda}+\frac{\bar{\lambda}}{K}\right)^{-1}$. Note that $\frac{c_{1}}{r_{1}}<1=\frac{c_{2}}{r_{2}}$, which is in agreement with the assumption that $c_{j} / r_{j}$ are non-decreasing in $j$.

For $K \geq 2$, since all the classes use only the first server,

$$
D_{K}\left(\boldsymbol{\lambda}^{=}, \mathbf{r}, \mathbf{c}\right)=c_{1} \frac{\bar{\lambda}}{r_{1}-\bar{\lambda}}=\bar{\lambda} \alpha\left(r_{1}\right)\left(r_{1}-\bar{\lambda}\right) .
$$

For $K=1$,

$$
D_{1}(\bar{\lambda}, \mathbf{r}, \mathbf{c})=\sum_{j \in \mathcal{S}^{*}(1)} c_{j} \frac{y_{j}(1)}{r_{j}-y_{j}(1)} .
$$

which, upon substituting from (42), gives

$$
D_{1}(\bar{\lambda}, \mathbf{r}, \mathbf{c})=\sum_{j \in \mathcal{S}^{*}(1)} c_{j}\left(\sqrt{\gamma(1) r_{j} / c_{j}}-1\right)=\sum_{j \in \mathcal{S}^{*}(1)}\left(\sqrt{\gamma(1)} \sqrt{c_{j} r_{j}}-c_{j}\right)
$$


Since the centralized scheme uses all the $S$ servers, from Proposition 10, $\gamma(1)$ is such that

$$
\sum_{j=1}^{S} \frac{r_{j}}{\sqrt{r_{j} / c_{j} \gamma(1)}}-\sum_{j=1}^{S} r_{j}+\bar{\lambda}=0
$$

which upon substitution in (48) gives

$$
D_{1}(\bar{\lambda}, \mathbf{r}, \mathbf{c})=\frac{\left(\sum_{j=1}^{S} \sqrt{c_{j} r_{j}}\right)^{2}}{\sum_{j=1}^{S} r_{j}-\bar{\lambda}}-\sum_{j=1}^{S} c_{j}
$$

Since $c_{2}=r_{2}=1$, we obtain

$$
\begin{aligned}
D_{1}(\bar{\lambda}, \mathbf{r}, \mathbf{c}) & =\frac{\left(\sqrt{c_{1} r_{1}}+S-1\right)^{2}}{r_{1}-\bar{\lambda}+S-1}-\left(c_{1}+S-1\right) \\
& =\frac{(S-1)\left(2 \sqrt{c_{1} r_{1}}-\left(r_{1}-\bar{\lambda}\right)\right)-c_{1}(S-1-\bar{\lambda})}{r_{1}-\bar{\lambda}+S-1} \\
& =\frac{(S-1)(r-\bar{\lambda})\left(2 \sqrt{r_{1} \alpha\left(r_{1}\right)}-1\right)-(r-\bar{\lambda})^{2} \alpha\left(r_{1}\right)(S-1-\bar{\lambda})}{r_{1}-\bar{\lambda}+S-1}
\end{aligned}
$$

From the above equation and (47),

$$
\frac{D_{K}\left(\boldsymbol{\lambda}^{=}, \mathbf{r}, \mathbf{c}\right)}{D_{1}(\bar{\lambda}, \mathbf{r}, \mathbf{c})}=\frac{\bar{\lambda} \alpha\left(r_{1}\right)}{\frac{(S-1)\left(2 \sqrt{r_{1} \alpha\left(r_{1}\right)}-1\right)-(r-\bar{\lambda}) \alpha\left(r_{1}\right)(S-1-\bar{\lambda})}{r_{1}-\bar{\lambda}+S-1}} .
$$

Taking the limit as $r_{1} \downarrow \bar{\lambda}$,

$$
\frac{D_{K}\left(\boldsymbol{\lambda}^{=}, \mathbf{r}, \mathbf{c}\right)}{D_{1}(\bar{\lambda}, \mathbf{r}, \mathbf{c})}=\frac{\bar{\lambda} \alpha(\bar{\lambda})}{2(\bar{\lambda} \alpha(\bar{\lambda}))^{1 / 2}-1}
$$

Note that the RHS in the above equation is increasing in $\bar{\lambda} \alpha(\bar{\lambda})$, and that $\bar{\lambda} \alpha(\bar{\lambda})$ has to be chosen in the interval $(1, K)$. Choosing the larger value, we obtain

$$
\frac{D_{K}\left(\boldsymbol{\lambda}^{=}, \mathbf{r}, \mathbf{c}\right)}{D_{1}(\bar{\lambda}, \mathbf{r}, \mathbf{c})}=\frac{K}{2 \sqrt{K}-1}
$$

which proves the inequality (13).

\subsection{Discussion on the PoA}

We first give the proof of Theorem 2 .

Proof of Theorem 2. From Propositions 12 and 13 we can conclude that

$$
\frac{K}{2 \sqrt{K}-1} \leq \operatorname{PoA}(K) \leq \sqrt{K} .
$$


We first note that the bounds on the PoA are valid for all values of $K$ and not just asymptotically. From these bounds, we can infer that the PoA grows as $\sqrt{K}$ as $K$ grows to infinity. Thus, the PoA can be made arbitrarily large in the limit $K \rightarrow \infty$, which is an alternative proof of Theorem 5 in [1] for the Wardrop equilibrium. In the other extreme case of $K=1$, the bounds lead to $\operatorname{PoA}(1)=1$, which is consistent with the fact that the case $K=1$ corresponds to the centralized setting.

We also observe that the PoA is independent of the number of servers the bounds are valid as long as there are at least two servers. This result is in contrast to the corresponding one for the case when server costs are equal, for which the PoA was shown to be bounded by the number of servers ([12], [21]) in the non-atomic game. Thus, we infer that the inclusion of unequal server costs has a non-negligible impact on the PoA in the sense that, even in a system with two servers, the PoA can be of the order of $\sqrt{K}$.

\section{Conclusions and future work}

We investigated the performance of non-cooperative load-balancing in processorsharing server-farms. We have first shown that the worst global performance is obtained when all $K$ dispatchers route exactly the same amount of traffic. This result implies that the analysis of the PoA can be done by focusing on the symmetric case, and therefore using the potential function method. We have then proved that, for a system with two or more servers, the PoA is lower bounded by $K /(2 \sqrt{K}-1)$ and upper bounded by $\sqrt{K}$, independently of the number of servers.

We believe that this methodology can be generalized to other network topologies than the parallel link scenario considered in this paper. We therefore plan to investigate under which conditions the symmetry of traffic demands leads to a maximum global cost for general network topologies.

\section{References}

[1] E. Altman, U. Ayesta, and B. J. Prabhu. Load balancing in processor sharing systems. To appear in Telecommunication Systems, 2009.

[2] C. H. Bell and S. Stidham. Individual versus social optimization in the allocation of customers to alternative servers. Management Science, 29:831839, 1983.

[3] S. C. Borst. Optimal probabilistic allocation of customer types to servers. In Proceedings of ACM SIGMETRICS, pages 116-125, Sept. 1995. 
[4] V. Cardellini, E. Casalicchio, M. Colajanni, and P. S. Yu. The state of the art in locally distributed web-server systems. ACM Computing Surveys, 34(2):263-311, 2001.

[5] H. L. Chen, J. Marden, and A. Wierman. The effect of local scheduling in load balancing designs. In Proceedings of IEEE Infocom, 2009.

[6] Y.-C. Chow and W. H. Kohler. Models for dynamic load balancing in a heterogeneous multiple processor system. IEEE Transactions on Computers, 28(5):354-361, 1979.

[7] R. Cominetti, J. R. Correa, and N. E. Stier-Moses. The impact of oligopolistic competition in networks. Operations Research, Published online in Articles in Advance, DOI: 10.1287/opre.1080.0653, June 2009.

[8] A. Czumaj, P. Krysta, and B. Vocking. Selfish traffic allocation for server farms. In Proceedings of STOC, 2002.

[9] S. El-Zoghdy, H. Kameda, and J. Li. Comparison of dynamic vs. static load-balancing policies in a mainframe-personal computer network model. Information, 5(4):431-446, 2002.

[10] H. Feng, V. Misra, and D. Rubenstein. Optimal state-free, size-aware dispatching for heterogeneous M/G/-type systems. Performance Evaluation, 62(1-4):36-39, 2005.

[11] V. Gupta, M. Harchol-Balter, K. Sigman, and W. Whitt. Analysis of jointhe-shortest-queue routing for web server farms. In Proceedings of Performance, 2007.

[12] M. Haviv and T. Roughgarden. The price of anarchy in an exponential multi-server. Operations Research Letters, 35:421-426, 2007.

[13] H. Kameda, J. Li, C. Kim, and Y. Zhang. Optimal load balancing in distributed computer systems. Springer-Verlag, 1997.

[14] Y. Korilis, A. Lazar, and A. Orda. Capacity allocation under noncooperative routing. IEEE Transactions on Automatic Control, 42(3):309-325, March 1997.

[15] E. Koutsoupias and C. H. Papadimitriou. Worst-case equilibria. In STACS 1999, 1999.

[16] D. Monderer and L. S. Shapley. Potential games. Games and Econ. Behavior, 14:124-143, 1996.

[17] L. M. Ni and K. Hwang. Optimal load balancing in a multiple processor with many job classes. IEEE Trans. Software Eng., 11(5):491-496, 1985. 
[18] A. Orda, R. Rom, and N. Shimkin. Competitive routing in multi-user communication networks. IEEE/ACM Transactions on Networking, 1:510521, October 1993.

[19] J. Rosen. Existence and uniqueness of equilibrium points for concave nperson games. Econometrica, 33:520-534, july 1965.

[20] B. Shirazi, A. Hurson, and K. Kavi. Scheduling and load-balancing in parallel and distributed systems. Silver Spring, MD: IEEE Computer Society Press, 1995.

[21] T. Wu and D. Starobinski. A comparative analysis of server selection in content replication networks. IEEE/ACM Trans. Netw., 16(6):1461-1474, 2008. 